\title{
GSK789: A Selective Inhibitor of the First Bromodomains (BD1) of the Bromo and Extra Terminal Domain (BET) Proteins
}

\section{Supporting Information}

Robert J. Watson ${ }^{1}$, Paul Bamborough*2, Heather Barnett ${ }^{3}$, Chun-wa Chung ${ }^{2}$, Rob Davis ${ }^{1}$, Laurie Gordon ${ }^{2}$, Paola Grandi ${ }^{4}$, Massimo Petretich ${ }^{4}$, Alex Phillipou ${ }^{2}$, Rab K. Prinjha ${ }^{1}$, Inmaculada Rioja ${ }^{1}$, Peter Soden ${ }^{1}$, Thilo Werner ${ }^{4}$,Emmanuel H. Demont*1.

${ }^{1}$ Epigenetics Discovery Performance Unit, ${ }^{2}$ Molecular Discovery Research, and ${ }^{3}$ Flexible Discovery Unit, GlaxoSmithKline Medicines Research Centre, Gunnels Wood Road, Stevenage, Hertfordshire, SG1 2NY, U.K. ${ }^{4}$ Cellzome GmbH, Molecular Discovery Research, GlaxoSmithKline, Meyerhofstrasse 1, 69117 Heidelberg, Germany.

\section{Table of Contents}

S2 - Table S1. BRD4 BD1 and BD2 and ATAD2 $\mathrm{pIC}_{50}$ s for exemplified compounds.

S3 - Table S2. BET BD1 and BD2 $\mathrm{pIC}_{50}$ s for exemplified compounds.

S4 - Table S3. Data collection and refinement statistics

S5 - Table S4. Selectivity profile of $\mathbf{4 2}$ in the BROMOscan panel.

S6 - Table S5. Comparison of TAF1 (BD2) GSK TR-FRET and BROMOscan assays.

S8 - Table S6. Activity of the enantiomer of $\mathbf{4 2}$ against BET bromodomains.

S9 - Figure S1. Sequence variation between BET bromodomains.

S10 - Figure S2. OMIT density maps for ligands.

S11 - Figure S3. Bromodomain tree showing BROMOscan selectivity profile of $\mathbf{4 2}$.

S12 - Figure S4. BIOmap profile of $\mathbf{4 2}$ and comparison with (+)-JQ1 and I-BET151.

S13 - 1H and 13C NMR spectrums of compound $\mathbf{4 2}$

S16 - LCMS traces of compounds disclosed 
Table S1. Full data for all compounds mentioned, including mean $\mathrm{pIC}_{50}$, standard deviation, and the number of independent test occasions in the BRD4 BD1 and BD2 assays and chromatographic LogD

\begin{tabular}{|c|c|c|c|c|}
\hline Cpd & $\begin{array}{c}\text { BRD4 BD1 TR-FRET } \\
\text { pIC }_{50}\end{array}$ & $\begin{array}{c}\text { BRD4 BD2 TR-FRET } \\
\text { pIC }_{50}\end{array}$ & $\begin{array}{c}\text { ATAD2 TR-FRET } \\
\text { pIC }_{50}\end{array}$ & $\begin{array}{c}\text { ChromLogD } \\
@ \text { pH } 7.4\end{array}$ \\
\hline 1 & $5.4+/-0.09(4)$ & $4.8+/-0.06(4)$ & $4.8+/-0.09(3)$ & 0.55 \\
\hline 2 & $6.1+/-0.03(4)$ & $5.4+/-0.02(4)$ & $4.5+/-0.09(3)$ & 2.51 \\
\hline 3 & $5.4+/-0.04(4)$ & $4.5+/-0.11(3 / 4)^{1}$ & $5.6(2)$ & 3.28 \\
\hline 4 & $5.6+/-0.1(5)$ & $4.8+/-0.12(5)$ & $5.7+/-0.02(2)$ & 1.18 \\
\hline 5 & $4.9+/-0.07(3)$ & $4.6+/-0.11(3)$ & $6+/-0.19(64)$ & 0.99 \\
\hline 6 & $4.8+/-0.15(10)$ & $5.3+/-0.65(7 / 9)^{1}$ & $6.9+/-0.09(5)$ & 1.62 \\
\hline 7 & $4.6+/-0.12(6)$ & $4.5(1 / 14)^{1}$ & $7.3+/-0.17(12)$ & 3.17 \\
\hline 8 & $6.1+/-0.05(5)$ & $<4.3(5)$ & $<4(2)$ & 2.03 \\
\hline 9 & $5.3+/-0.07(2)$ & $<4.3(2)$ & $4.2+/-0.02(2)$ & 1.88 \\
\hline 10 & $5.8+/-0.11(2)$ & $<4.3(2)$ & $<4(2)$ & 2.36 \\
\hline 11 & $5.7+/-0.13(2)$ & $<4.3(2)$ & $<4(2)$ & 1.6 \\
\hline 12 & $5.6+/-0.06(2)$ & $4.4(1 / 2)$ & $<4(2)$ & 1.87 \\
\hline 13 & $5.5+/-0.02(2)$ & $<4.3(2)$ & $4.1+/-0.01(2)$ & 1.05 \\
\hline 14 & $5.3+/-0.19(2)$ & $<4.3(3)$ & $<4(2)$ & 1.89 \\
\hline 15 & $5.6+/-0.07(2)$ & $4.9+/-0.05(2)$ & $<4(2)$ & 0.71 \\
\hline 16 & $5.4+/-0.06(2)$ & $<4.3(2)$ & $<4(2)$ & 0.85 \\
\hline 17 & $4.6+/-0.18(2)$ & $<4.3(2)$ & $<4(2)$ & 0.98 \\
\hline 18 & $5.1+/-0.35(2)$ & $4.5+/-0.14(2)$ & $4.4+/-0.01(1 / 2)$ & 1.08 \\
\hline 27 & $6.2+/-0.13(2)$ & $4.6+/-0.11(2)$ & & 3.36 \\
\hline 28 & $5.9+/-0.06(2)$ & $<4.3(2)$ & & 2.51 \\
\hline 29 & $7.3+/-0.02(2)$ & $4.5+/-0.11(2)$ & & 3.23 \\
\hline 30 & $7+/-0.09(2)$ & $<4.3(2)$ & & 3.79 \\
\hline 31 & $7.4+/-0.1(2)$ & $4.6+/-0.07(2)$ & & 3.14 \\
\hline 32 & $7.7+/-0.1(2)$ & $4.9+/-0.15(2)$ & & 3.12 \\
\hline 33 & 5.8 & $5(1)$ & $5.2(1)$ & 3.12 \\
\hline 34 & $7.4+/-0.03(2)$ & $4.9+/-0.05(2)$ & & 2.91 \\
\hline 35 & $6.8+/-0.01(2)$ & $4.8+/-0.03(2)$ & & 1.47 \\
\hline 36 & $6.9+/-0.02(2)$ & $4.5(1)$ & & 3.55 \\
\hline 37 & $6.7+/-0.04(2)$ & $4.4+/-0.08(2)$ & & 4.4 \\
\hline 38 & $6+/-0.07(2)$ & $4.9+/-0.11(2)$ & & 4.78 \\
\hline 39 & $5.9+/-0.06(2)$ & $4.4(1 / 2)$ & & 2.07 \\
\hline 40 & $5.5+/-0.02(3)$ & $4.5+/-0.2(2)$ & & 2.52 \\
\hline 41 & $6.5+/-0.12(4)$ & $4.8+/-.05(4)$ & & 3.66 \\
\hline 42 & $7.5+/-0.26(4)$ & $4.5+/-0.09(2 / 4)$ & & 3.99 \\
\hline 43 & $7.6+/-0.03(2)$ & $4.8+/-0.04(2)$ & & 4.33 \\
\hline 44 & $7.4+/-0.01(2)$ & $4.7+/-0.08(2)$ & & 4.46 \\
\hline 45 & $7.3+/-0.04(2)$ & $4.8(1 / 2)$ & & 4.35 \\
\hline 46 & $7.8+/-0.04(2)$ & $5.4+/-0.01(2)$ & & 4.61 \\
\hline 47 & $7.2+/-0(2)$ & $<4.3(2)$ & & 5.3 \\
\hline 48 & $7.3+/-0.03(2)$ & $4.8+/-0.05(2)$ & & 5.27 \\
\hline 49 & $7+/-0.04(2)$ & $4.5+/-0.06(2)$ & & 5.13 \\
\hline 50 & $7.1+/-0.06(2)$ & $4.7+/-0.18(2)$ & & 2.43 \\
\hline
\end{tabular}

${ }^{1}$ Some $\mathrm{pIC}_{50}$ results below the curve-fitting threshold could not be included in mean and SD. In these cases, the number included in mean / number of times tested are both shown. 
Table S2. All measured $\mathrm{plC}_{50} \mathrm{~S}$ on BD1 and BD2 for all four BET proteins for all compounds mentioned

\begin{tabular}{|c|c|c|c|c|c|c|c|c|}
\hline \multirow{2}{*}{ Cpd } & \multicolumn{3}{|c|}{ BD1 TR-FRET pIC50 } & \multicolumn{3}{c|}{ BD2 TR-FRET pIC50 } \\
\cline { 2 - 9 } & BRD4 & BRD3 & BRD2 & BRDT & BRD4 & BRD3 & BRD2 & BRDT \\
\hline 1 & 5.4 & & & & 4.8 & & & \\
\hline 2 & 6.1 & & & & 5.4 & & & \\
\hline 3 & 5.4 & 5.1 & 5.1 & 4.8 & 4.5 & 4.3 & $<4.3$ & $<4.3$ \\
\hline 4 & 5.6 & 5.1 & 5.2 & 5.1 & 4.8 & 4.7 & 4.7 & 4.5 \\
\hline 5 & 4.9 & 4.6 & 4.6 & 4.8 & 4.6 & 4.9 & 4.8 & 4.4 \\
\hline 6 & 4.8 & $<4.3$ & 4.5 & 4.5 & 5.3 & $<4.3$ & $<4.3$ & $<4.3$ \\
\hline 7 & 4.6 & $<4.3$ & 4.4 & 4.3 & 4.5 & $<4.3$ & $<4.3$ & $<4.3$ \\
\hline 8 & 6.1 & 6.3 & 6.1 & 5.5 & $<4.3$ & 4.5 & 4.9 & $<4.3$ \\
\hline 9 & 5.3 & & 5.4 & 5 & $<4.3$ & & 4.8 & 4.6 \\
\hline 10 & 5.8 & 5.8 & 5.6 & 5.1 & $<4.3$ & 4.7 & 5.2 & 5 \\
\hline 11 & 5.7 & 5.4 & 5.3 & 4.8 & $<4.3$ & $<4.3$ & 5.3 & 5.4 \\
\hline 12 & 5.6 & 5.5 & 5.6 & 4.9 & 4.4 & 4.7 & & 4.4 \\
\hline 13 & 5.5 & 5.4 & 5.5 & 4.8 & $<4.3$ & $<4.3$ & 4.7 & 4.6 \\
\hline 14 & 5.3 & 5 & 5.2 & 4.8 & $<4.3$ & $<4.3$ & 5.1 & 5 \\
\hline 15 & 5.6 & 5.4 & 5.4 & 4.8 & 4.9 & 4.9 & 5.1 & 4.8 \\
\hline 16 & 5.4 & 5.3 & 5.3 & 4.8 & $<4.3$ & $<4.3$ & 4.8 & 4.8 \\
\hline 17 & 4.6 & 4.4 & 4.7 & $<4.3$ & $<4.3$ & $<4.3$ & $<4.3$ & $<4.3$ \\
\hline 18 & 5.1 & 4.9 & 5 & 4.6 & 4.5 & $<4.3$ & 4.7 & 4.6 \\
\hline 27 & 6.2 & 6.4 & 6.2 & 5.9 & 4.6 & 4.8 & 4.8 & 4.6 \\
\hline 28 & 5.9 & 5.9 & 5.9 & 5.4 & $<4.3$ & 4.4 & 4.7 & $<4.3$ \\
\hline 29 & 7.3 & 7.2 & 7.1 & 6.7 & 4.5 & 4.5 & $<4.3$ & 4.5 \\
\hline 30 & 7 & & & & $<4.3$ & & & \\
\hline 31 & 7.4 & 6.6 & 6.7 & 6 & 4.6 & 4.6 & 5.3 & 5.2 \\
\hline 32 & 7.7 & 6.4 & 7 & 6.4 & 4.9 & 4.6 & 5.1 & 5.1 \\
\hline 33 & 5.8 & 5.9 & 6 & 5.1 & 5 & 4.9 & 4.9 & 4.9 \\
\hline 34 & 7.4 & & & & 4.9 & & & \\
\hline 35 & 6.8 & 6.9 & 6.7 & 6.1 & 4.8 & 4.9 & 4.5 & 4.6 \\
\hline 36 & 6.9 & 6.8 & 6.9 & 6.4 & 4.5 & 4.5 & $<4.3$ & $<4.3$ \\
\hline 37 & 6.7 & 4.5 & 7.2 & 6.2 & 4.4 & 4.3 & & $<4.3$ \\
\hline 38 & 6 & 6.4 & & 5.5 & 4.9 & 4.7 & $<4.3$ & $<4.3$ \\
\hline 39 & 5.9 & 5.9 & 5.8 & 5.2 & 4.4 & 4.6 & $<4.3$ & $<4.3$ \\
\hline 40 & 5.5 & 5.4 & 5.6 & 5 & 4.5 & 4.6 & 4.8 & $<4.3$ \\
\hline 41 & 6.5 & 6.4 & 6.3 & 5.6 & 4.8 & 4.9 & 5 & 4.6 \\
\hline 42 & 7.5 & 6.8 & 7 & 6.2 & 4.5 & 4.7 & 4.7 & 4.9 \\
\hline 43 & 7.6 & 7.4 & 7.1 & 6.6 & 4.8 & 5 & 4.5 & 4.7 \\
\hline 44 & 7.4 & 6.9 & 6.5 & 6.1 & 4.7 & 4.9 & 4.5 & 4.6 \\
\hline 45 & 7.3 & 6.6 & 6.4 & 5.9 & 4.8 & 4.8 & 4.7 & 4.5 \\
\hline 46 & 7.8 & 7.4 & 7.1 & 6.7 & 5.4 & $<4.3$ & 4.4 & 4.4 \\
\hline 47 & 7.2 & 6.7 & 6.6 & 6.2 & $<4.3$ & 5.3 & 5.1 & 4.9 \\
\hline 48 & 7.3 & 7.1 & 7.2 & 6.7 & 4.8 & 5.2 & 4.9 & 4.7 \\
\hline 49 & 7 & & & & 4.5 & & & \\
\hline 50 & 7.1 & 7.1 & 6.8 & 6.4 & 4.7 & 5 & 4.8 & 4.8 \\
\hline & & & & & & & & \\
\hline
\end{tabular}


Table S3: Data collection and refinement statistics

\begin{tabular}{|c|c|c|}
\hline (collection on a single crystal) & BRD4 BD1 / compound 8 & BRD4 BD1 / compound 42 \\
\hline \multicolumn{3}{|l|}{ Data collection } \\
\hline Space group & $\mathrm{P} 22_{1} 2_{1} 2_{1}$ & $\mathrm{P} 2{ }_{1} 2_{1} 2_{1}$ \\
\hline \multicolumn{3}{|l|}{ Cell dimensions } \\
\hline$a, b, c(\AA)$ & $43.430,48.980,61.240$ & $43.968,48.286,61.245$ \\
\hline$\alpha, \beta, \gamma\left(^{\circ}\right)$ & $90.000,90.000,90.000$ & $90.000,90.000,90.000$ \\
\hline Resolution ( $\AA$ ) & $16.66-1.26(1.29-1.26)$ & $37.92-1.62(1.71-1.62)$ \\
\hline$R_{\text {merge }}$ & $0.043(0.551)$ & $0.041(0.331)$ \\
\hline $\mathrm{CC}(1 / 2)$ & $0.999(0.818)$ & $0.997(0.414)$ \\
\hline$l / \sigma l$ & $27.4(2.5)$ & $17.5(2.7)$ \\
\hline Completeness (\%) & $99.1(95.5)$ & $91.7(76.2)$ \\
\hline Redundancy & $6.2(5.1)$ & $2.7(1.5)$ \\
\hline \multicolumn{3}{|l|}{ Refinement } \\
\hline Resolution $(\AA ̊)$ & $16.66-1.26$ & $37.92-1.62$ \\
\hline No. reflections & 222718 (12483) & $41460(2796)$ \\
\hline No. uniq reflections & $35636(2463)$ & $15543(1831)$ \\
\hline$R_{\text {work/ }} R_{\text {free }}$ & $0.196 / 0.214$ & $0.183 / 0.224$ \\
\hline No. atoms & 1353 & 1327 \\
\hline Protein & 1067 & 1069 \\
\hline Ligand/ion & $28 / 0$ & $34 / 0$ \\
\hline Water & 258 & 224 \\
\hline \multicolumn{3}{|l|}{ B-factors } \\
\hline Protein & 19.58 & 18.42 \\
\hline Ligand/ion & 16.99 & 15.63 \\
\hline Water & 35.31 & 31.49 \\
\hline \multicolumn{3}{|l|}{ R.m.s deviations } \\
\hline Bond lengths ( $(\AA)$ & 0.003 & 0.004 \\
\hline Bond angles (ㅇ) & 1.159 & 1.213 \\
\hline
\end{tabular}

*Highest resolution shell is shown in parenthesis 
Table S4. Selectivity profile of compound $\mathbf{4 2}$ in the BROMOscan panel (DiscoveRx Corp).

$\Delta$ is the selectivity of compound $\mathbf{4 2}$ expressed in log units for BRD4 BD1 over each bromodomain.

\begin{tabular}{|c|c|c|}
\hline Bromodomain & pKd & $\Delta$ \\
\hline ATAD2A & 4.8 & $>2.9$ \\
\hline ATAD2B & 4.8 & $>2.9$ \\
\hline BAZ2A & 4.8 & $>2.9$ \\
\hline BAZ2B & $<4.5$ & $>3.2$ \\
\hline BRD1 & $<4.5$ & $>3.2$ \\
\hline BRD2 (BD1) & 7.7 & 0 \\
\hline BRD2 (BD2) & 4.6 & 3.1 \\
\hline BRD3 (BD1) & 7.8 & -0.1 \\
\hline BRD3 (BD2) & 4.7 & 3 \\
\hline BRD4 (BD1) & 7.7 & 0 \\
\hline BRD4 (BD2) & 4.5 & 3.2 \\
\hline BRD7 & $<4.6$ & $>3.1$ \\
\hline BRD8 (BD1) & $<4.5$ & $>3.2$ \\
\hline BRD8 (BD2) & $<4.5$ & $>3.2$ \\
\hline BRD9 & $<4.5$ & $>3.2$ \\
\hline BRDT (BD1) & 7.7 & 0 \\
\hline BRDT $(1,2)$ & 7 & 0.7 \\
\hline BRDT (BD2) & 4.7 & 3 \\
\hline BRPF1 & $<4.5$ & $>3.2$ \\
\hline BRPF3 & $<4.5$ & $>3.2$ \\
\hline CECR2 & $<4.5$ & $>3.2$ \\
\hline CREBBP & $<4.5$ & $>3.2$ \\
\hline EP300 & 4.6 & 3.1 \\
\hline FALZ & $<4.5$ & 3.2 \\
\hline GCN5L2 & $<4.5$ & $>3.2$ \\
\hline PBRM1 (BD2) & $<4.5$ & $>3.2$ \\
\hline PBRM1(5) & 5 & 2.7 \\
\hline PCAF & $<4.5$ & $>3.2$ \\
\hline SMARCA2 & $<4.5$ & $>3.2$ \\
\hline SMARCA4 & $<4.5$ & $>3.2$ \\
\hline TAF1 (BD2) & 7.3 & 0.4 \\
\hline TAF1L (BD2) & 6.4 & 1.3 \\
\hline TRIM24 (TIF1 $\alpha)(B D)$ & 4.7 & 3.0 \\
\hline TRIM24 (TIF1 $\alpha)$ (PHD \& BD) & $<4.5$ & $>3.2$ \\
\hline TRIM33 (TIF1 $\gamma)$ (PHD \& BD) & $<4.5$ & $>3.2$ \\
\hline WDR9 (BD2) & $<4.5$ & $>3.2$ \\
\hline
\end{tabular}


Table S5. TAF1 (BD2) inhibition data for all compounds in GSK collection screened both in GSK TR-FRET assay and BROMOscan assay (DiscoveRx Corp.). Compound $\mathbf{4 2}$ is entry $\mathbf{7 4}$

\begin{tabular}{|c|c|c|}
\hline Entry & TAF1 (BD2) DiscoveRx plC 50 & $\begin{array}{c}\text { TAF1(BD2) TR-FRET } \\
\text { pIC }_{50}\end{array}$ \\
\hline 1 & $<4.5$ & $<4(4)$ \\
\hline 2 & $<4.5$ & $<4(1)$ \\
\hline 3 & $<4.5$ & $4.1(1 / 5)^{1}$ \\
\hline 4 & $<4.5$ & $<4(2)$ \\
\hline 5 & $<4.5$ & $<4(2)$ \\
\hline 6 & $<4.5$ & $<5(1)$ \\
\hline 7 & $<4.5$ & $<4(3)$ \\
\hline 8 & $<5$ & $4(1 / 3)^{1}$ \\
\hline 9 & $<5$ & $5.8+/-0.15(3)$ \\
\hline 10 & $<5.3$ & $4.1(1 / 2)^{1}$ \\
\hline 11 & $<5.3$ & $<4(3)$ \\
\hline 12 & 4.6 & $<4(4)$ \\
\hline 13 & 4.7 & $<5(1)$ \\
\hline 14 & 4.8 & $<4(1)$ \\
\hline 15 & 4.8 & $<5(1)$ \\
\hline 16 & 5 & $4(2 / 3)^{1}$ \\
\hline 17 & 5.1 & $<4(3)$ \\
\hline 18 & 5.1 & $4(1 / 3)$ \\
\hline 19 & 5.1 & $4.2(1 / 3)^{1}$ \\
\hline 20 & 5.2 & $<4(3)$ \\
\hline 21 & 5.2 & $4.1+/-0.04(3)$ \\
\hline 22 & 5.2 & $<4(3)$ \\
\hline 23 & 5.3 & $4.2(1 / 3)^{1}$ \\
\hline 24 & 5.3 & $<5(1)$ \\
\hline 25 & 5.3 & $<4(3)$ \\
\hline 26 & 5.3 & $4.5+/-0.12(3)$ \\
\hline 27 & 5.4 & $<4(3)$ \\
\hline 28 & 5.4 & $4.2+/-0.03(2 / 4)^{1}$ \\
\hline 29 & 5.4 & $4(2 / 4)$ \\
\hline 30 & 5.4 & $<4(3)$ \\
\hline 31 & 5.5 & $<4(4)$ \\
\hline 32 & 5.5 & $4.6+/-0.15(3)$ \\
\hline 33 & 5.7 & $<4(3)$ \\
\hline 34 & 5.7 & $4.4+/-0.06(8)$ \\
\hline 35 & 5.8 & $4.6+/-0.07(3)$ \\
\hline 36 & 5.9 & $4.3+/-0.07(3)$ \\
\hline 37 & $5.9+/-0.11(4)$ & $4.8+/-0.03(3)$ \\
\hline 38 & $6+/-0.12(4)$ & $4.5+/-0.04(3)$ \\
\hline 39 & 6.1 & $5.1+/-0.05(3)$ \\
\hline 40 & 6.1 & $4.9+/-0.08(3)$ \\
\hline 41 & 6.1 & $5+/-0.07(3)$ \\
\hline 42 & 6.2 & $4.8+/-0.06(3)$ \\
\hline 43 & 6.2 & $4.7+/-0.07(3)$ \\
\hline
\end{tabular}




\begin{tabular}{|c|c|c|}
\hline 44 & 6.2 & $5.4+/-0.07(3)$ \\
\hline 45 & 6.2 & $5.3+/-0.06(3)$ \\
\hline 46 & 6.3 & $4.1+/-0.11(3)$ \\
\hline 47 & 6.4 & $5+/-0.09(4)$ \\
\hline 48 & 6.5 & $5.1+/-0.07(3)$ \\
\hline 49 & 6.5 & $<4(3)$ \\
\hline 50 & 6.5 & $5.6(1)$ \\
\hline 51 & 6.6 & $4.3+/-0.04(2)$ \\
\hline 52 & 6.6 & $5.1+/-0.01(3)$ \\
\hline 53 & 6.6 & $5.4+/-0.02(3)$ \\
\hline 54 & 6.6 & $5.1+/-0.05(3)$ \\
\hline 55 & 6.7 & $<4(3)$ \\
\hline 56 & 6.7 & $<4(2)$ \\
\hline 57 & 6.7 & $5.6+/-0.06(3)$ \\
\hline 58 & 6.7 & $4.9+/-0.14(3)$ \\
\hline 59 & 6.7 & $5.6+/-0.08(3)$ \\
\hline 60 & 6.7 & $5.8+/-0.04(3)$ \\
\hline 61 & 6.8 & $<5(1)$ \\
\hline 62 & 6.8 & $6+/-0.07(3)$ \\
\hline 63 & 6.8 & $5.3+/-0.13(3)$ \\
\hline 64 & 6.8 & $5.2+/-0.04(3)$ \\
\hline 65 & $6.9+/-0.2(4)$ & $6+/-0.02(3)$ \\
\hline 66 & 7.1 & $5.5+/-0.22(3)$ \\
\hline 67 & 7.1 & $5.7+/-0.05(3)$ \\
\hline 68 & 7.2 & $6.3+/-0.06(3)$ \\
\hline 69 & 7.3 & $6(1)$ \\
\hline 70 & 7.3 & $5.9+/-0.09(3)$ \\
\hline 71 & 7.3 & $6.1+/-0.04(3)$ \\
\hline 72 & 7.3 & $6.1+/-0.07(3)$ \\
\hline 73 & 7.3 & $6.1+/-0.01(3)$ \\
\hline 74 & $7.3+/-0.19(4)$ & $5+/-0.09(3)$ \\
\hline 75 & 7.6 & $6+/-0.15(3)$ \\
\hline 76 & 7.8 & $6.3+/-0.1(5)$ \\
\hline 77 & 8.5 & $6.5+/-0.11(3)$ \\
\hline 78 & 8.9 & $6.3+/-0.04(2)$ \\
\hline 79 & 8.9 & $6.8+/-0.07(2)$ \\
\hline 80 & 9 & $7.4+/-0.04(3)$ \\
\hline 81 & 9.1 & $6.9+/-0.04(3)$ \\
\hline 82 & 9.2 & $7.3+/-0.19(5)$ \\
\hline 83 & 9.2 & $6.8+/-0.08(3)$ \\
\hline 84 & 9.2 & $7.4+/-0.23(3)$ \\
\hline 85 & 9.4 & $7.5+/-0.29(5)$ \\
\hline 86 & 9.7 & $7.1+/-0.16(3)$ \\
\hline 87 & 9.7 & $7.4+/-0.04(3)$ \\
\hline
\end{tabular}

${ }^{1}$ Some $\mathrm{pIC}_{50}$ results below the curve-fitting threshold could not be included in mean and SD. In these cases, the number included in mean / number of times tested are both shown 
Table S6. Activity of the enantiomer of compound 42 GSK789 against the bromodomains of the BET family (TR-FRET data).

\begin{tabular}{|c|c|c|c|c|c|c|c|}
\hline \multicolumn{4}{|c|}{ BD1 } & \multicolumn{4}{c|}{ BD2 } \\
\hline BRD4 & BRD3 & BRD2 & BRDT & BRD4 & BRD3 & BRD2 & BRDT \\
\hline $\begin{array}{c}5.8+/-0.14 \\
(4)\end{array}$ & $5.4(2)$ & $\begin{array}{c}5.6+/-0.01 \\
(2)\end{array}$ & $\begin{array}{c}5.2+/-0.01 \\
(2)\end{array}$ & $\begin{array}{c}4.5+/-0.14 \\
(3 / 4)^{1}\end{array}$ & $\begin{array}{c}4.7+/-0.09 \\
(2)\end{array}$ & $4.8(1)$ & $\begin{array}{c}5.1+/-0.03 \\
(2)\end{array}$ \\
\hline
\end{tabular}

${ }^{1}$ Some $\mathrm{plC}_{50}$ results below the curve-fitting threshold could not be included in mean and SD. In these cases, the number included in mean / number of times tested are both shown 
Figure S1. Homology between human BET BD1 and BD2 domains. (A) Sequence alignment. (B) Percent sequence identity between BET family bromodomains over the domain (left) and the residues of the acetyl-lysine site (right). (C) Key residues in the BC loop of the eight BET bromodomains.

A)

BRD2 BD1 69 PKKPGRVTNQLLYLHKVVMKALWK---HQFAWTFRQPVDAVKLGLPDYHKIIKQPMD BRD3 BD1 29 PSKPGRKTNQLQYMQNVVVKTLWK---HQFAWPFYQPVDAIKLNLPDYHKIIKNPMD BRD4 BD1 53 PNKPKRQTNQLQYLLRVVLKTLWK---HQFAWPFQQPVDAVKLNLPDYYKIIKTPMD BRDT BD1 22 TKKNGRLTNQLQYLQKVVLKDLWK---HSFSWPFQRPVDAVKLKLPDYYTIIKNPMD BRD2 BD2 339 QSSKKGKLSEQLKHCNGILKELLSKKHAAYAWPFYKPVDASALGLHDYHDI IKHPMD BRD3 BD2 301 HAGKKGKLSEHLRYCDSILREMLSKKHAAYAWP FYKPVDAEALELHDYHDIIKHPMD BRD4 BD2 343 APEKSSKVSEQLKCCSGILKEMFAKKHAAYAWPFYKPVDVEALGLHDYCDIIKHPMD BRDT BD2 262 NVVKTVKVTEQLRHCSEILKEMLAKKHFSYAWPFYNPVDVNALGLHNYYDVVKNPMD
$\alpha A$
$\alpha B$
$B C$ loop
$\alpha C$

BRD2 BD1 123 MGTIKRRLENNYYWAASECMQDFNTMFTNCYIYNKPTDDIVLMAQTLEKIFLQKVAS BRD3 BD1 83 MGTIKKRLENNYYWSASECMQDFNTMFTNCYIYNKPTDDIVLMAQALEKIFLQKVAQ BRD4 BD1 107 MGTIKKRLENNYYWNAQECIQDFNTMFTNCY IYNKPGDDIVLMAEALEKLFLQKINE BRDT BD1 76 LNTIKKRLENKYYAKASECIEDFNTMF SNCYLYNKPGDDIVLMAQALEKLFMQKLSQ BRD2 BD2 396 LSTVKRKMENRDYRDAQEFAADVRLMF SNCYKYNPPDHDVVAMARKLQDVFEFRYAK BRD3 BD2 358 LSTVKRKMDGREYPDAQGFAADVRLMF SNCYKYNPPDHEVVAMARKLQDVFEMRFAK BRD4 BD2 400 MSTIKSKLEAREYRDAQEFGADVRLMF SNCYKYNPPDHEVVAMARKLQDVFEMRFAK BRDT BD2 319 LGTIKEKMDNQEYKDAYKFAADVRLMFMNCYKYNPPDHEVVTMARMLQDVFETHFSK it KAC-binding residue $\nabla$ BD1 Lys/BD2 Pro O BD1 Asp / BD2 His

B)

\begin{tabular}{|c|c|c|c|c|c|c|c|c|c|c|c|c|c|c|c|c|c|c|c|}
\hline \multirow{2}{*}{\multicolumn{2}{|c|}{$\begin{array}{c}\text { Domain } \\
\% \text { ID }\end{array}$}} & \multicolumn{4}{|c|}{ BD1 } & \multicolumn{4}{|c|}{ BD2 } & \multirow{2}{*}{\multicolumn{2}{|c|}{$\begin{array}{c}\text { KAc Site } \\
\text { \%ID }\end{array}$}} & \multicolumn{4}{|c|}{ BD1 } & \multicolumn{4}{|c|}{ BD2 } \\
\hline & & BRD2 & BRD3 & BRD4 & BRDT & BRD2 & BRD3 & BRD4 & BRDT & & & BRD2 & BRD3 & BRD4 & BRDT & BRD2 & BRD3 & BRD4 & BRDT \\
\hline \multirow{4}{*}{ ㅁ } & BRD2 & 100 & 87 & 78 & 72 & 45 & 39 & 43 & 36 & \multirow{4}{*}{ దે } & BRD2 & 100 & 92 & 88 & 85 & 77 & 69 & 65 & 65 \\
\hline & BRD3 & 87 & 100 & 80 & 73 & 45 & 40 & 43 & 38 & & BRD3 & 92 & 100 & 92 & 85 & 77 & 73 & 65 & 65 \\
\hline & BRD4 & 78 & 80 & 100 & 72 & 43 & 37 & 42 & 37 & & BRD4 & 88 & 92 & 100 & 92 & 69 & 65 & 62 & 65 \\
\hline & BRDT & 72 & 73 & 72 & 100 & 43 & 37 & 40 & 43 & & BRDT & 85 & 85 & 92 & 100 & 69 & 65 & 62 & 65 \\
\hline \multirow{4}{*}{ ్ㅗ } & BRD2 & 45 & 45 & 43 & 43 & 100 & 84 & 83 & 68 & \multirow{4}{*}{ กิ } & BRD2 & 77 & 77 & 69 & 69 & 100 & 92 & 88 & 85 \\
\hline & BRD3 & 39 & 40 & 37 & 37 & 84 & 100 & 81 & 70 & & BRD3 & 69 & 73 & 65 & 65 & 92 & 100 & 88 & 85 \\
\hline & BRD4 & 43 & 43 & 42 & 40 & 83 & 81 & 100 & 70 & & BRD4 & 65 & 65 & 62 & 62 & 88 & 88 & 100 & 92 \\
\hline & BRDT & 36 & 38 & 37 & 43 & 68 & 70 & 70 & 100 & & BRDT & 65 & 65 & 65 & 65 & 85 & 85 & 92 & 100 \\
\hline
\end{tabular}

C)

\begin{tabular}{|l|cccccc|}
\hline & KAC N & BD1 K / BD2 P & \multicolumn{3}{c|}{ BD1 D / BD2 H } \\
\hline BRD2 & N156 & K157 & P158 & T159 & D160 & D161 \\
BRD3 & N116 & K117 & P118 & T119 & D120 & D121 \\
BRD4 & N140 & K141 & P142 & G143 & D144 & D145 \\
BRDT & N109 & K110 & P111 & G112 & D113 & D114 \\
\cline { 2 - 6 } BRD2 & N429 & P430 & P431 & D432 & H433 & D434 \\
BRD3 & N391 & P392 & P393 & D394 & H395 & E396 \\
BRD4 & N433 & P434 & P435 & D436 & H437 & E438 \\
BRDT & N352 & P353 & P354 & D355 & H356 & E357 \\
\hline
\end{tabular}


Figure S2. OMIT density maps for ligands.

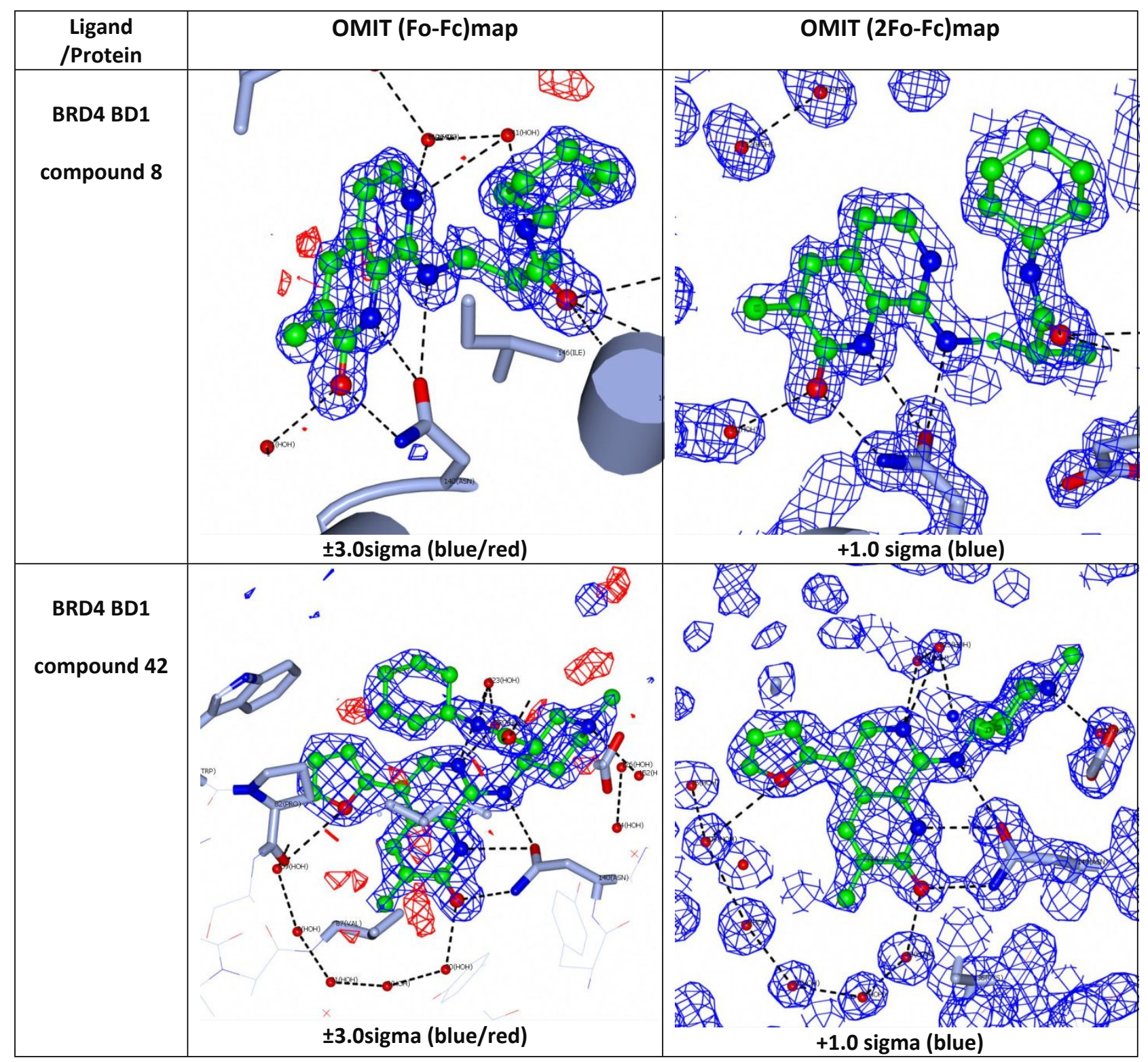


Figure S3. Fold selectivity of BRD4 BD2 against other bromodomfrom BROMOscan $\mathrm{K}_{\mathrm{d}}$ data.

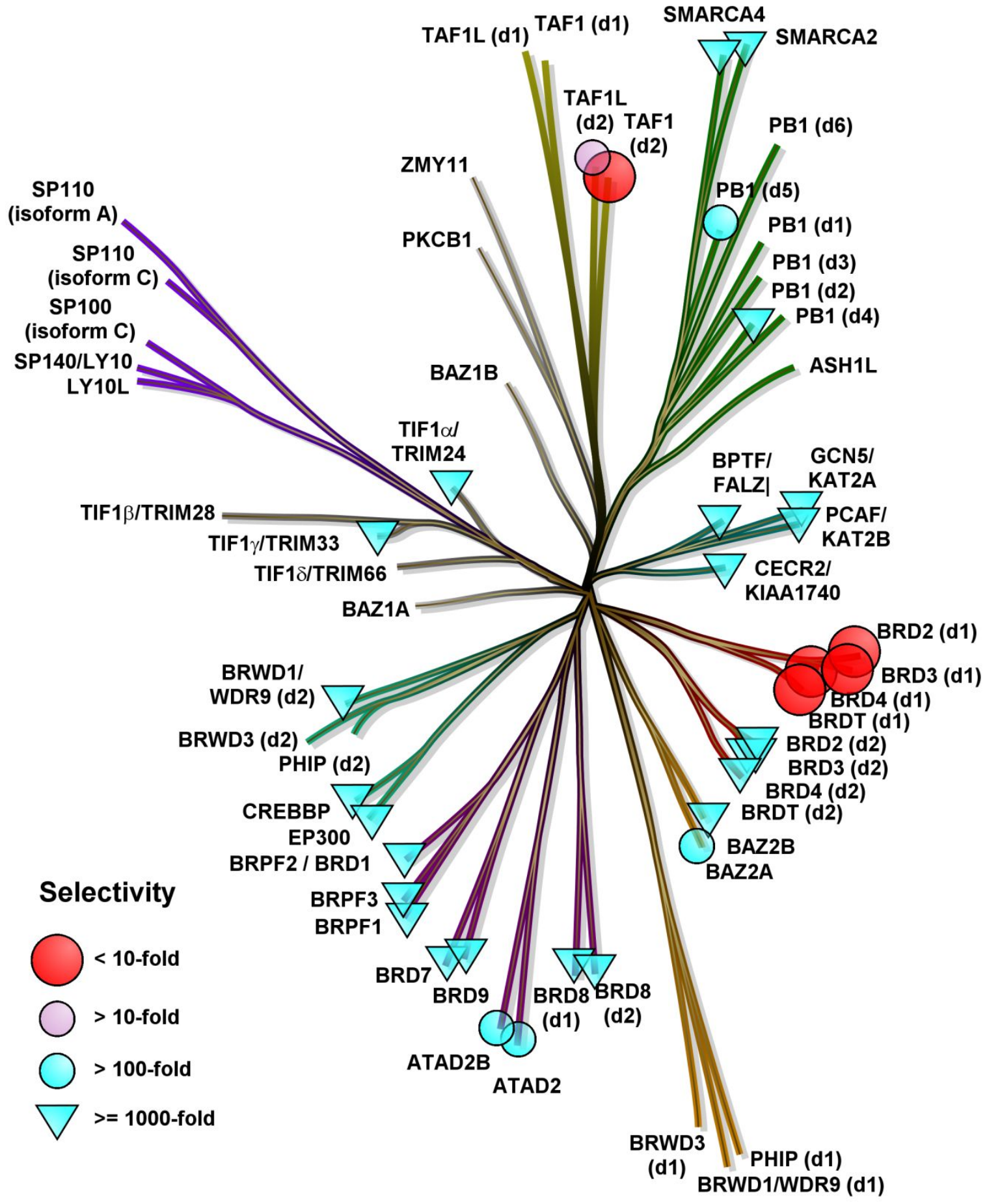


Figure S4. Overlay of the BioMAP profiles of GSK789 and pan BET inhibitors (+)-JQ1 and I-BET151. (A) BioMAP profile of GSK789 (10 $\mu$ M, red line) compared to the profile of (+)-JQ1 (370 nM, blue line). Common biomarker readouts are annotated when the readout for both profiles is outside of the significance envelope with an effect size $>20 \%$ ( $\mid \log 10$ ratio $\mid>0.1)$ in the same direction. (B) Overlay of the BioMAP profiles of GSK789 $(10 \mu M$, red line) and I-BET151 (3.3 $\mathrm{MM}$, black line) demonstrating similar phenotypic fingerprint. Differentiating biomarkers are annotated when one profile has a readout outside of the significance envelope with an effect size $>20 \%(\mid \log 10$ ratio $\mid>0.1)$, and the readout for the other profile is either inside the envelope or in the opposite direction.

A)

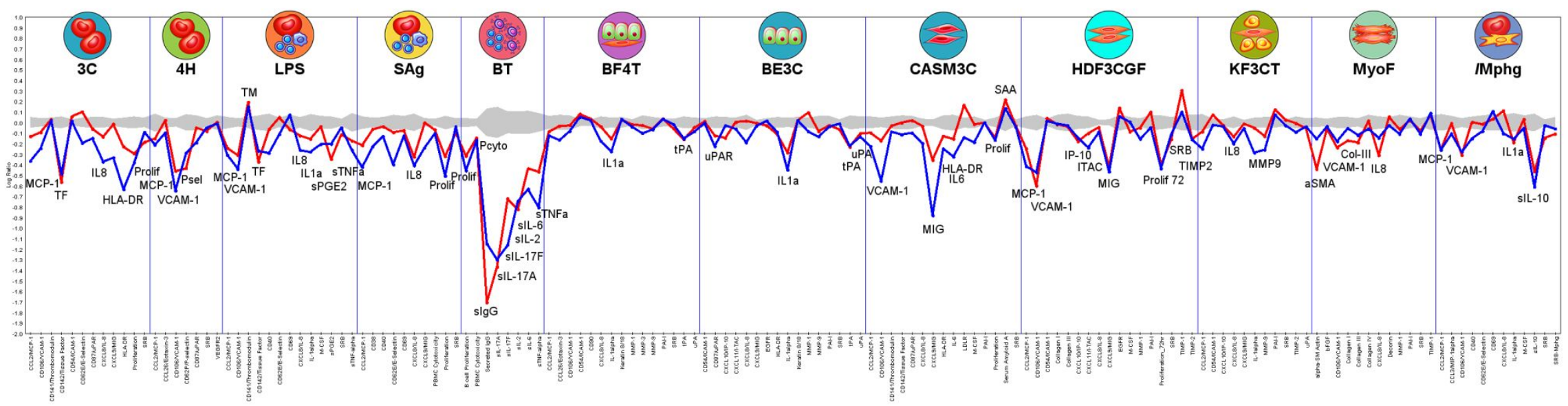

B)

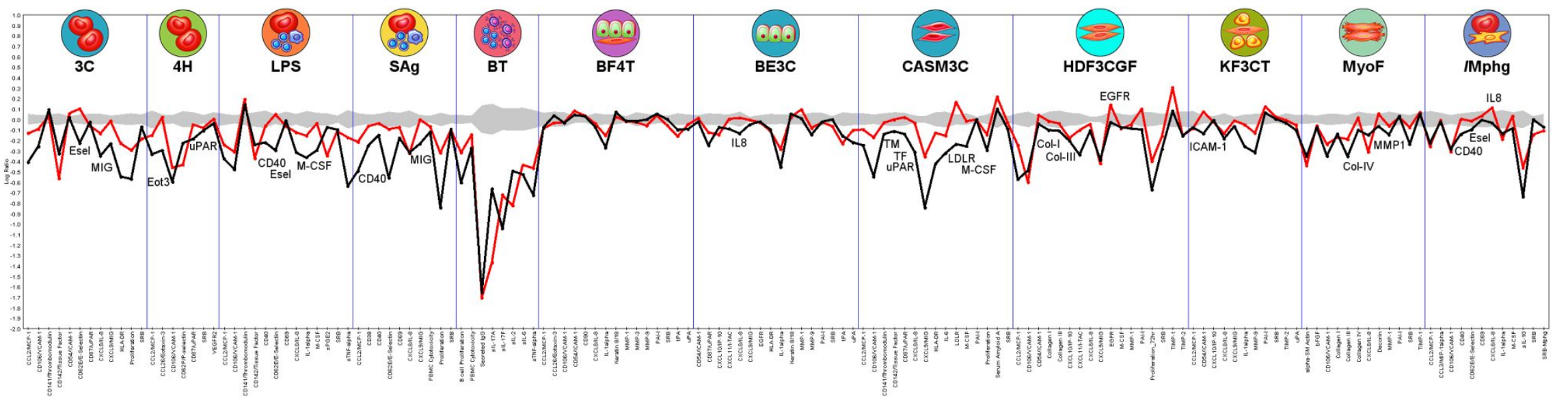




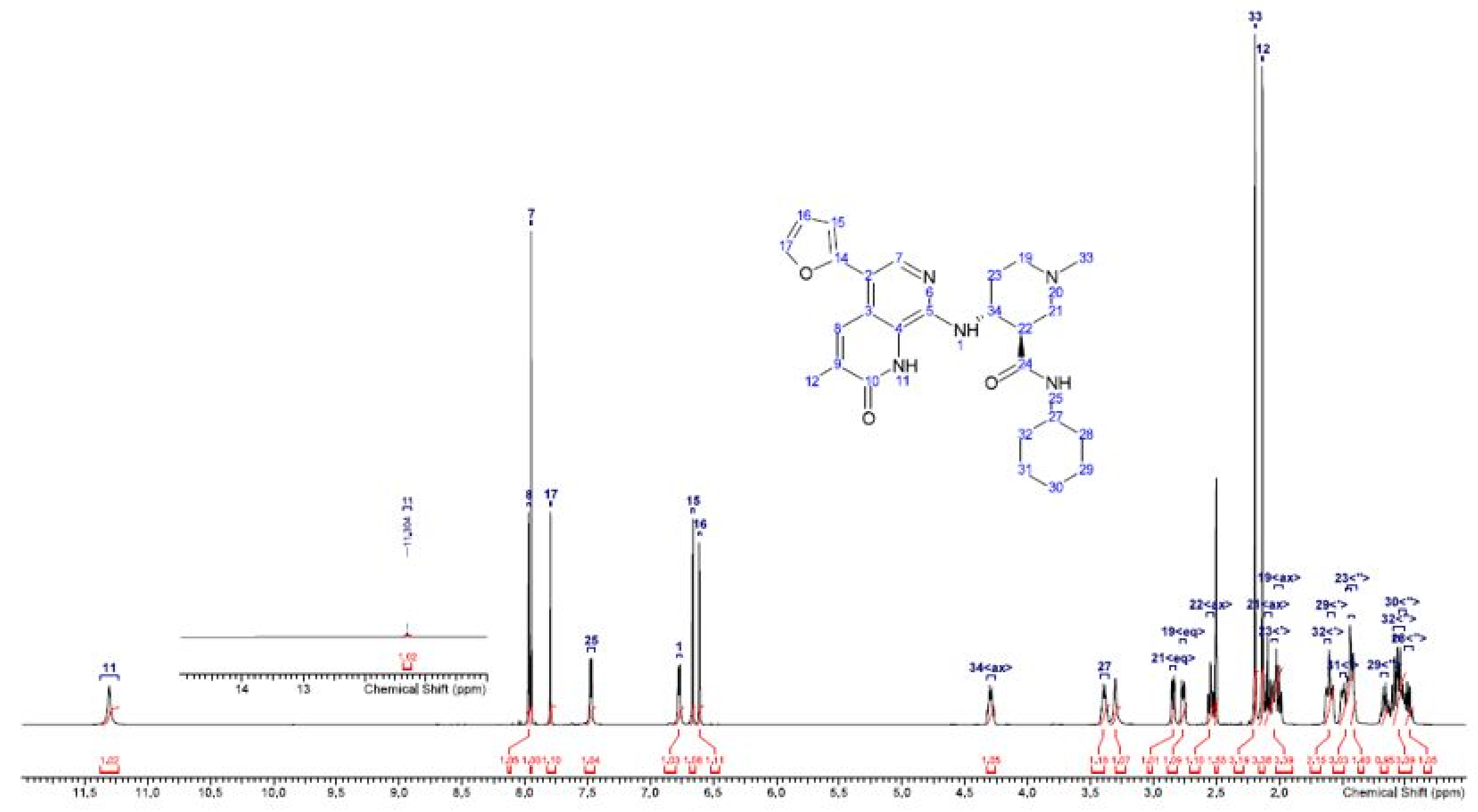




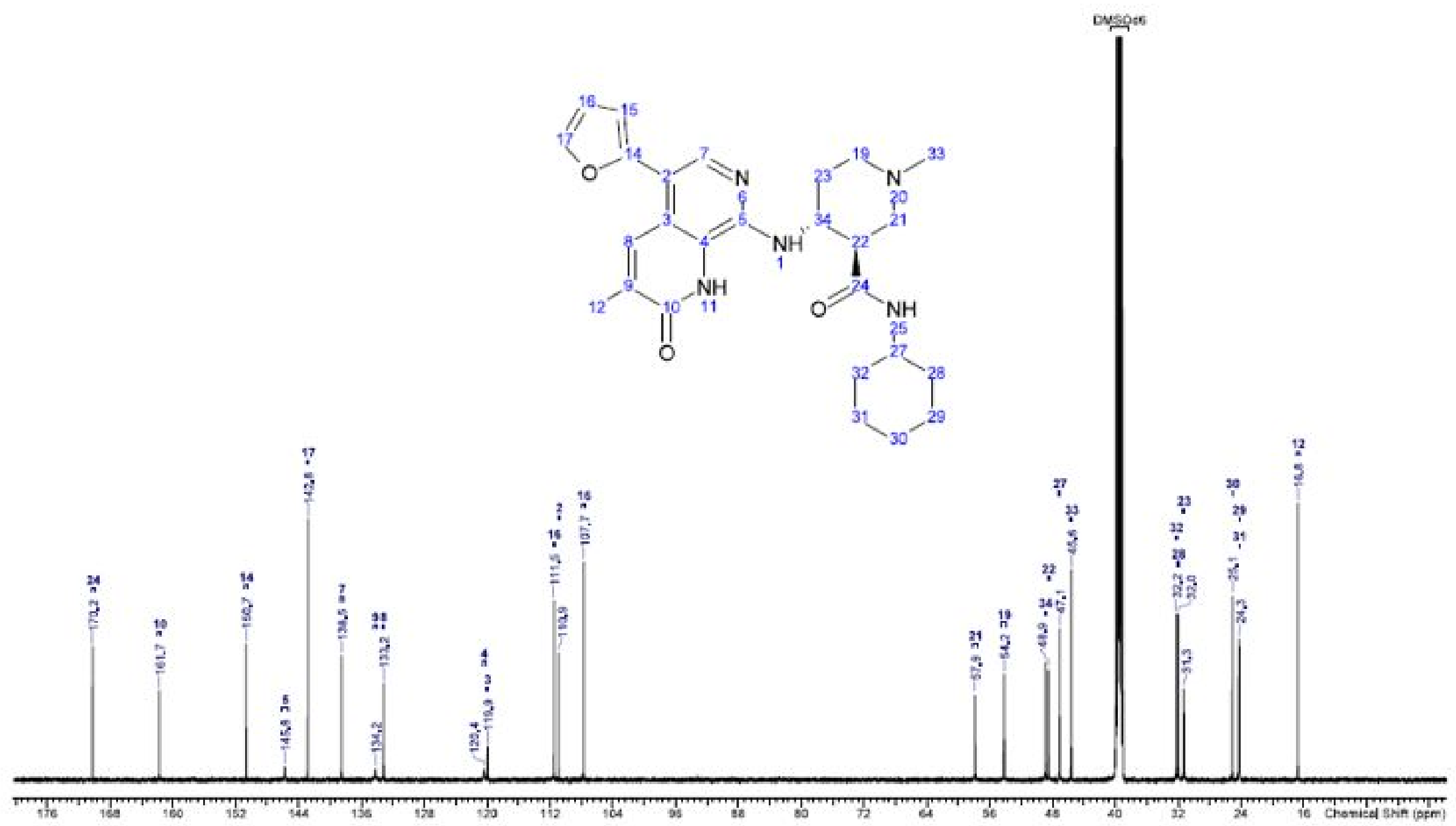




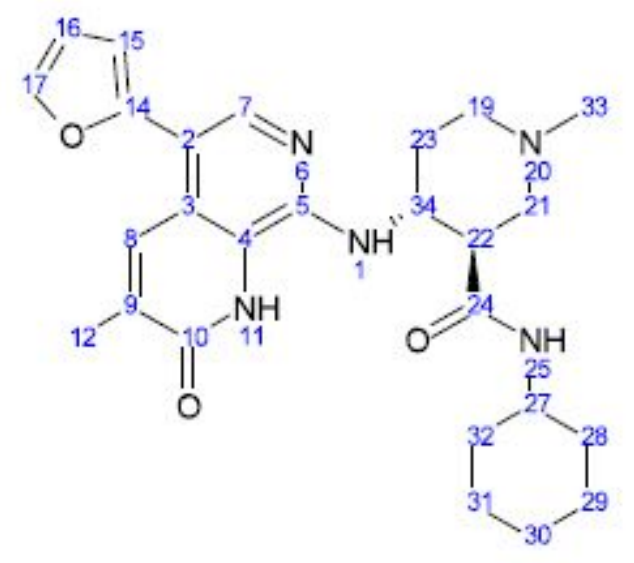

1H NMR (DMSO-d6,600MHz): $\delta 11.30$ (br s, 1H, H-11), 7.97 (q, $J=1.3 \mathrm{~Hz}, 1 \mathrm{H}, \mathrm{H}-8$ ), 7.95 (s, 1H, H-7), 7.80 (dd, J=2.0, 0.7 Hz, 1H, H-17), 7.47 (br d, $J=8.0 \mathrm{~Hz}, 1 \mathrm{H}, \mathrm{H}-25), 6.77$ (br d, $J=7.8 \mathrm{~Hz}, 1 \mathrm{H}, \mathrm{H}-1), 6.66$ (dd, $J=3.3,0.7 \mathrm{~Hz}, 1 \mathrm{H}, \mathrm{H}-15), 6.61$ (dd, $J=3.3,2.0 \mathrm{~Hz}, 1 \mathrm{H}, \mathrm{H}-16), 4.26-4.33$ (m, $1 \mathrm{H}, \mathrm{H}-34$ <ax>), $3.36-3.43(\mathrm{~m}, 1 \mathrm{H}, \mathrm{H}-27), 2.82-2.86(\mathrm{~m}, 1 \mathrm{H}, \mathrm{H}-21<\mathrm{eq}>), 2.76(\mathrm{br} \mathrm{d}, J=11.4 \mathrm{~Hz}, 1 \mathrm{H}, \mathrm{H}-19<\mathrm{eq}>), 2.54(\mathrm{td}, J=10.9,3.9 \mathrm{~Hz}, 1 \mathrm{H}, \mathrm{H}-22<\mathrm{ax}>$ ), $2.19(\mathrm{~s}, 3 \mathrm{H}, \mathrm{H}-33), 2.14(\mathrm{~d}, J=1.1 \mathrm{~Hz}, 3 \mathrm{H}, \mathrm{H}-12), 2.06-2.12(\mathrm{~m}, 1 \mathrm{H}, \mathrm{H}-21<\mathrm{ax}>), 2.02-2.07\left(\mathrm{~m}, 1 \mathrm{H}, \mathrm{H}-23<<^{\prime}>\right), 1.97-2.04(\mathrm{~m}, 1 \mathrm{H}, \mathrm{H}-19<\mathrm{ax}>), 1.59-$ $1.64\left(\mathrm{~m}, 1 \mathrm{H}, \mathrm{H}-32<^{\prime}>\right)$ ), $1.56-1.61\left(\mathrm{~m}, 1 \mathrm{H}, \mathrm{H}-29<^{\prime}>\right), 1.47-1.52\left(\mathrm{~m}, 1 \mathrm{H}, \mathrm{H}-31<^{\prime}>\right), 1.43-1.48\left(\mathrm{~m}, 1 \mathrm{H}, \mathrm{H}-30<^{\prime}>\right), 1.40-1.45\left(\mathrm{~m}, 1 \mathrm{H}, \mathrm{H}-28<^{\prime}>\right), 1.38-$ $1.46\left(m, 1 \mathrm{H}, \mathrm{H}-23<^{\prime \prime}>\right), 1.14-1.19(\mathrm{~m}, 1 \mathrm{H}, \mathrm{H}-29<">), 1.05-1.09(\mathrm{~m}, 1 \mathrm{H}, \mathrm{H}-31<">), 1.01-1.09(\mathrm{~m}, 1 \mathrm{H}, \mathrm{H}-32<">), 1.00-1.05(\mathrm{~m}, 1 \mathrm{H}, \mathrm{H}-30<">)$, $0.93-1.01(\mathrm{~m}, 1 \mathrm{H}, \mathrm{H}-28<">)$

${ }_{13}$ C NMR (DMSO-d , 151MHz): $\delta 170.2$ (s, C-24), 161.7 (s, C-10), 150.7 (s, C-14), 145.8 (br s, C-5), 142.8 (s, C-17), 138.5 (s, C-7), 134.2 (br s, C-9), 133.2 (s, C-8), 120.4 (br s, C-4), 119.9 (br s, C-3), 111.5 (s, C-16), 110.9 (s, C-2), 107.7 (s, C-15), 57.9 (s, C-21), 54.2 (s, C-19), 48.9 (s, C-34), 48.5 (s, C-22), 47.1 (s, C-27), 45.6 (s, C-33), 32.2 (s, C-32), 32.0 (s, C-28), 31.3 (s, C-23), 25.1 (s, C-30), 24.3 (br s, C-29), 24.3 (br s, C-31), 16.8 (s, C-12) 
LCMS cpd 8:

MS ES+ : $384.2321 .0000 \mathrm{Da}$

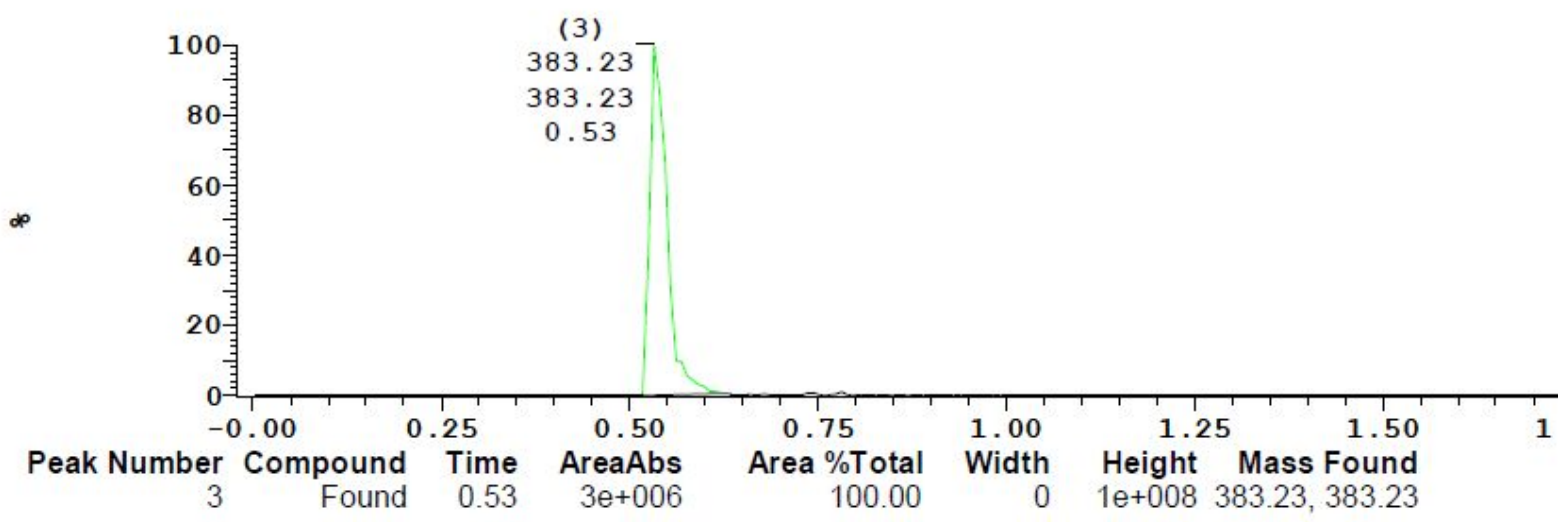

LCMS Cpd 9:

MS ES+ :384.232 1.0000Da

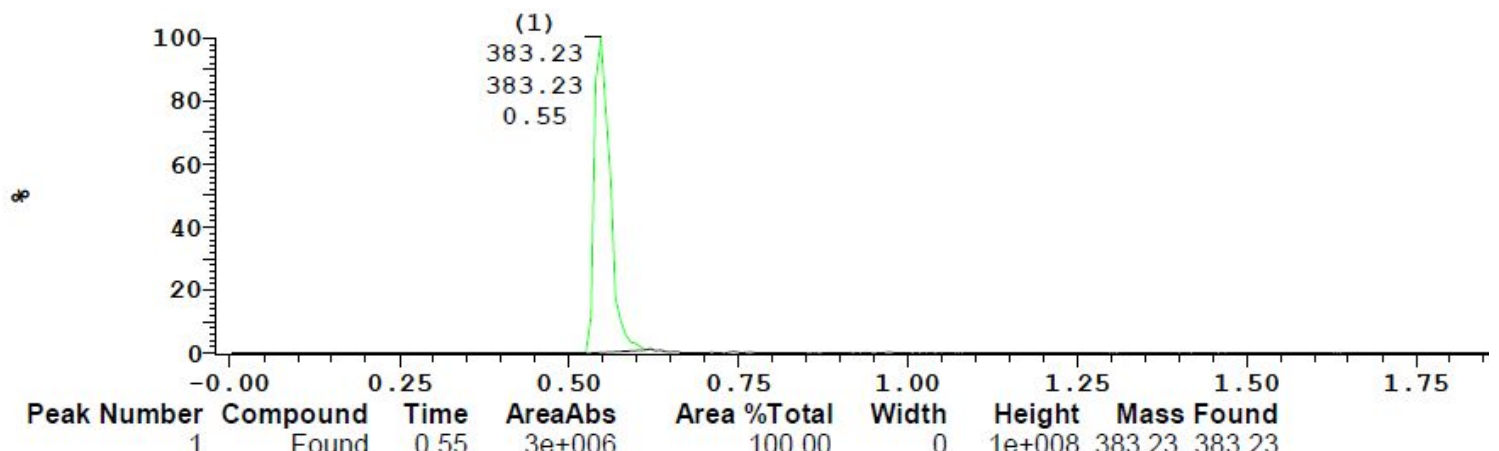


LCMS Cpd 10:

MS ES+: $384.2321 .0000 \mathrm{Da}$

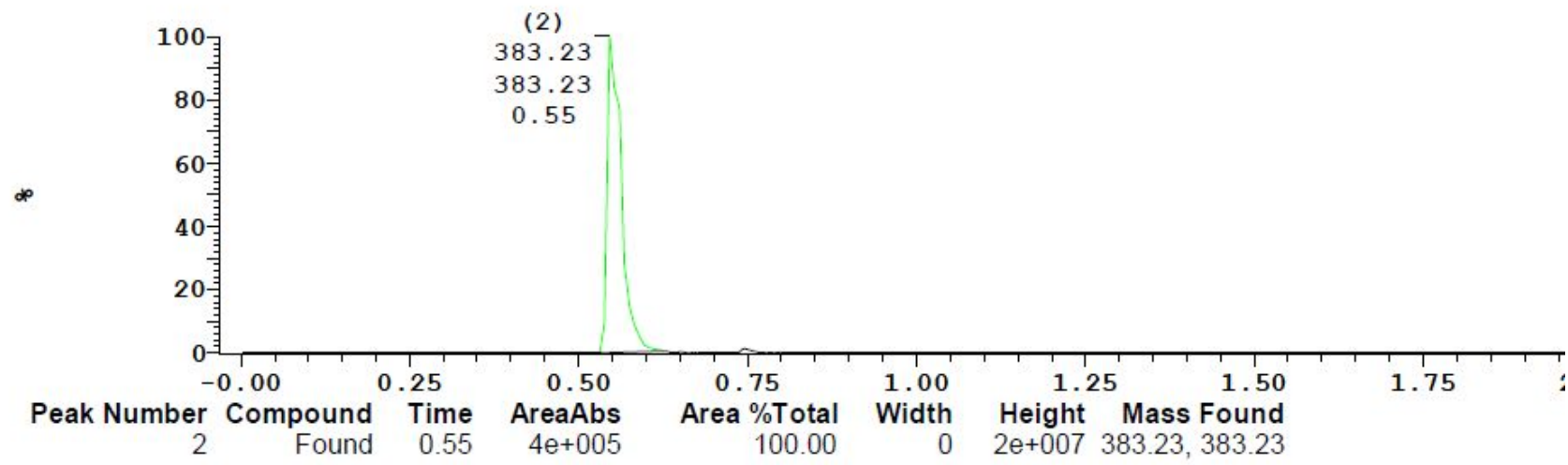

LCMS Cpd 11:

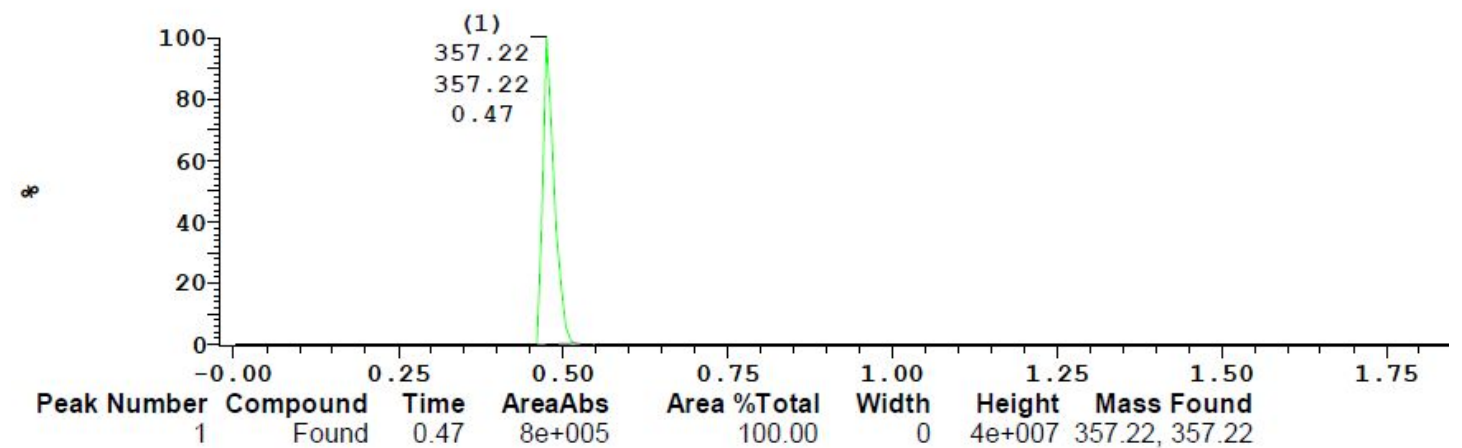


LCMS Cpd 12:

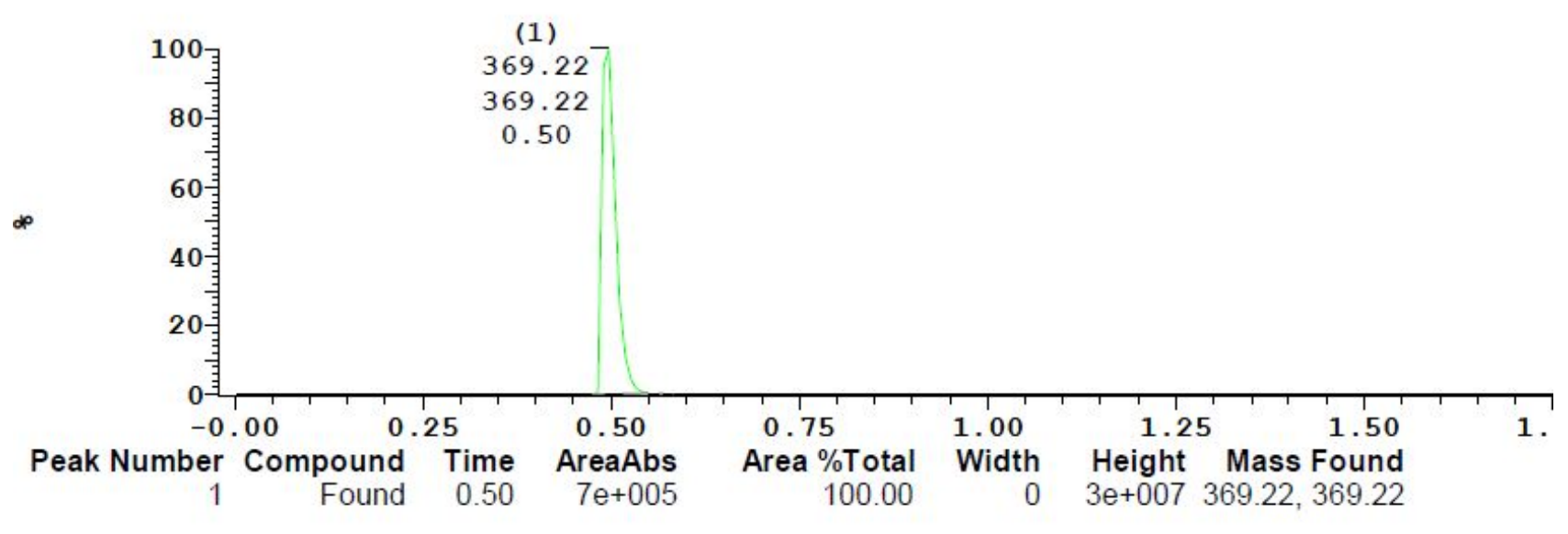

LCMS Cpd 13:

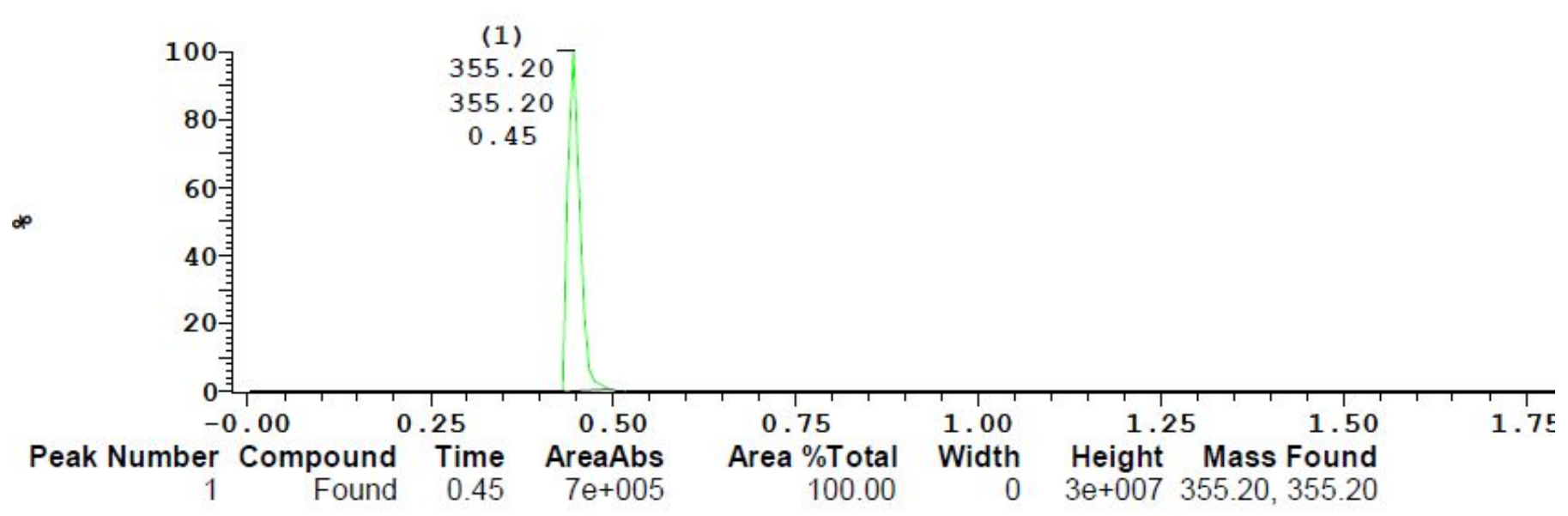


LCMS Cpd 14:

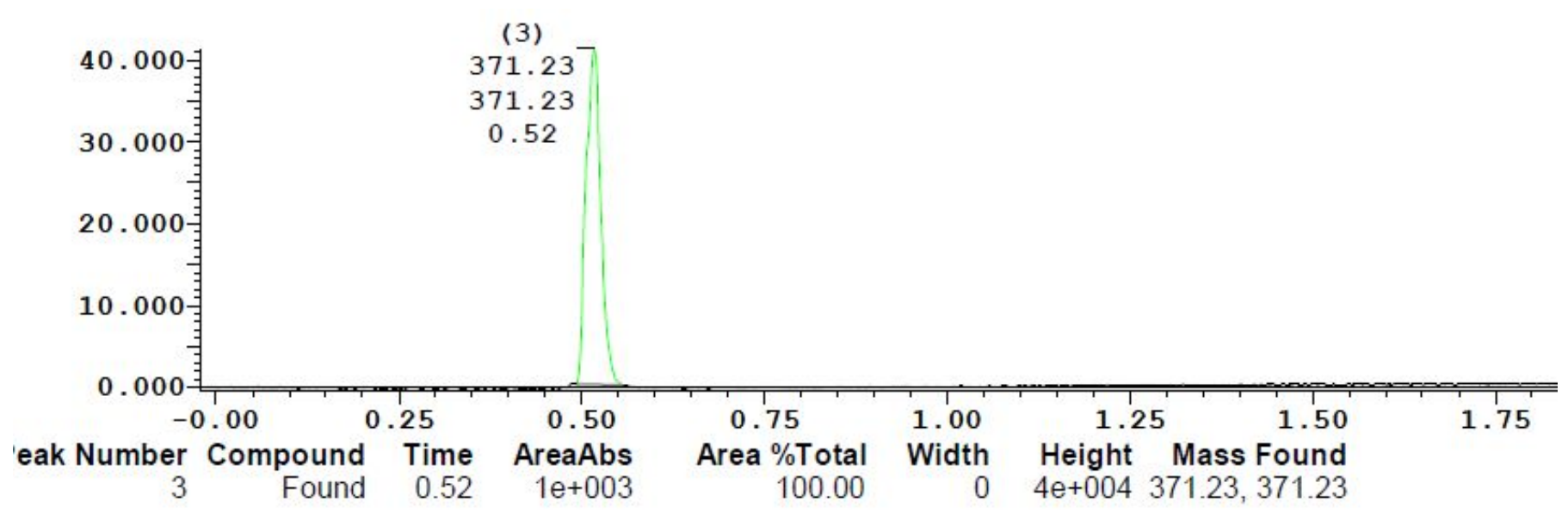

LCMS Cpd 15:

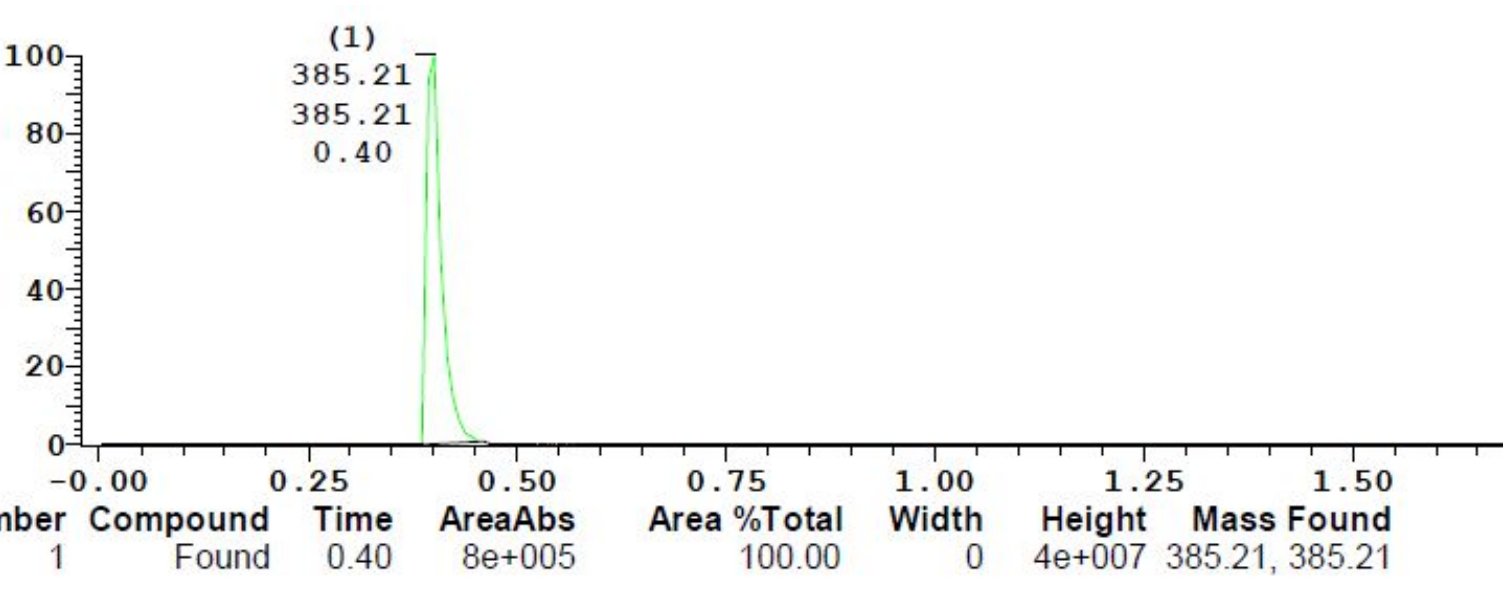

- .284 211 1 กnกกn- 
LCMS Cpd 16:

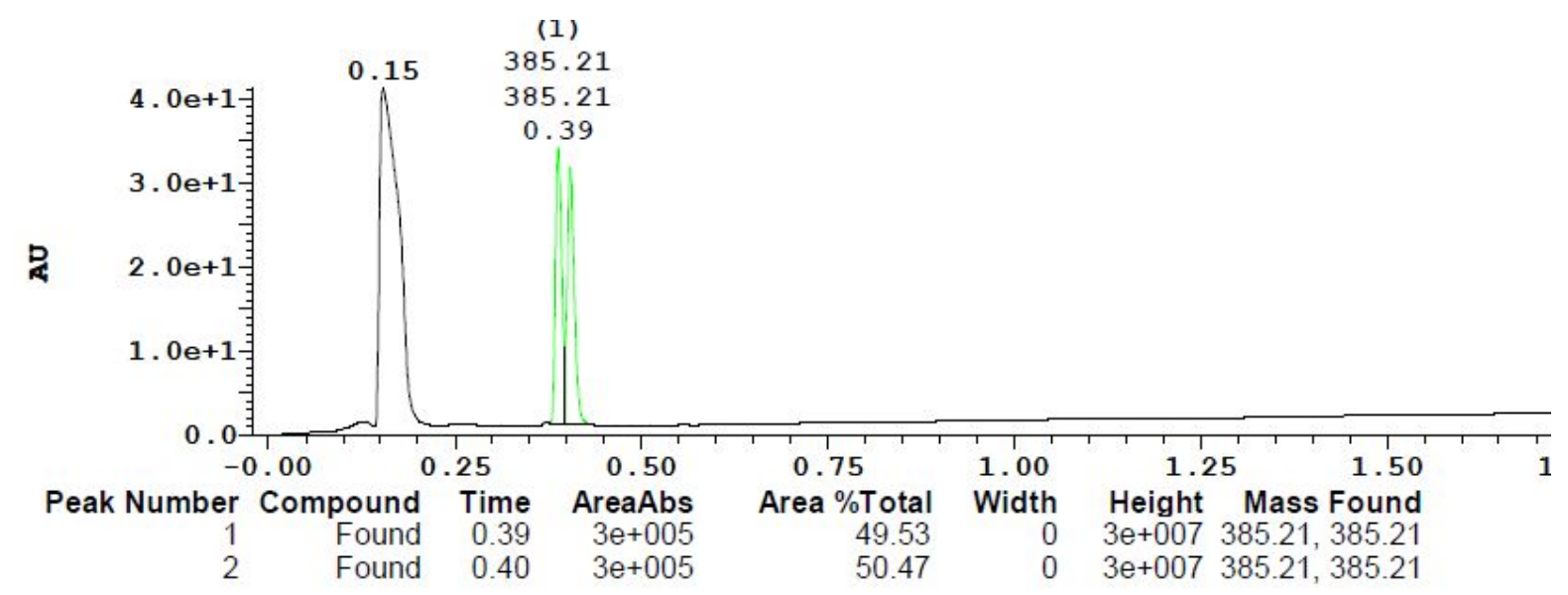

\section{LCMS Cpd 17:}

MS ES+ :386.211 $1.0000 \mathrm{Da}$

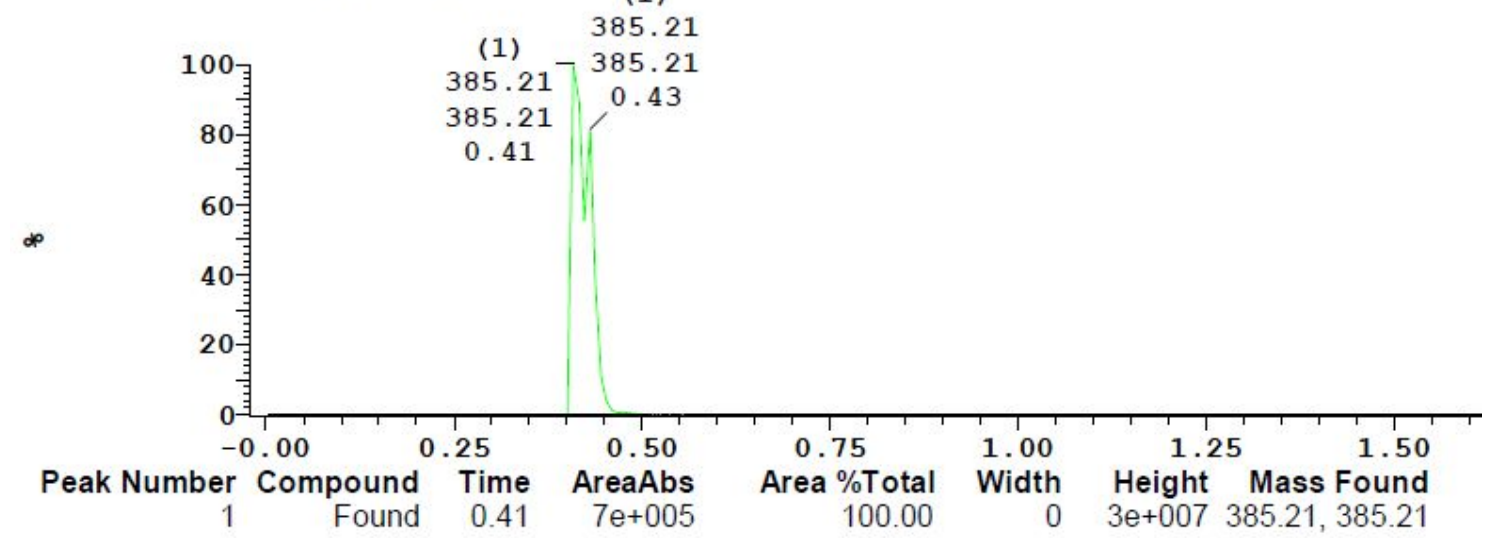




\section{LCMS Cpd18:}

MS ES+ :374.211 1.0000Da

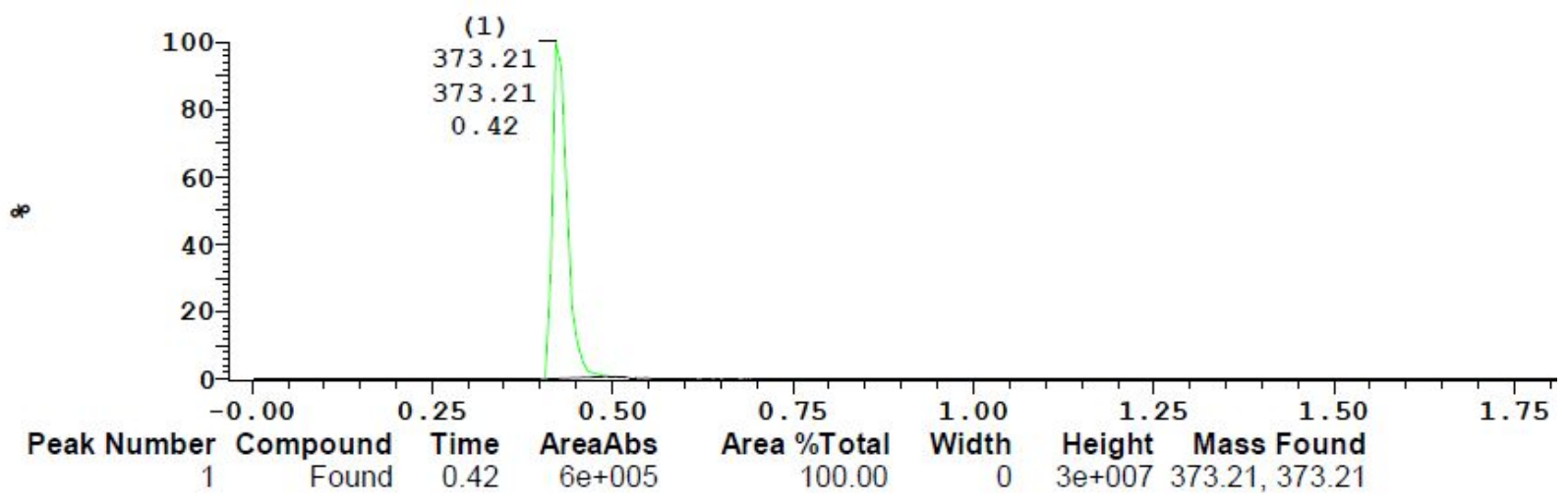

LCMS Cpd 27:

UV Detector: TIC

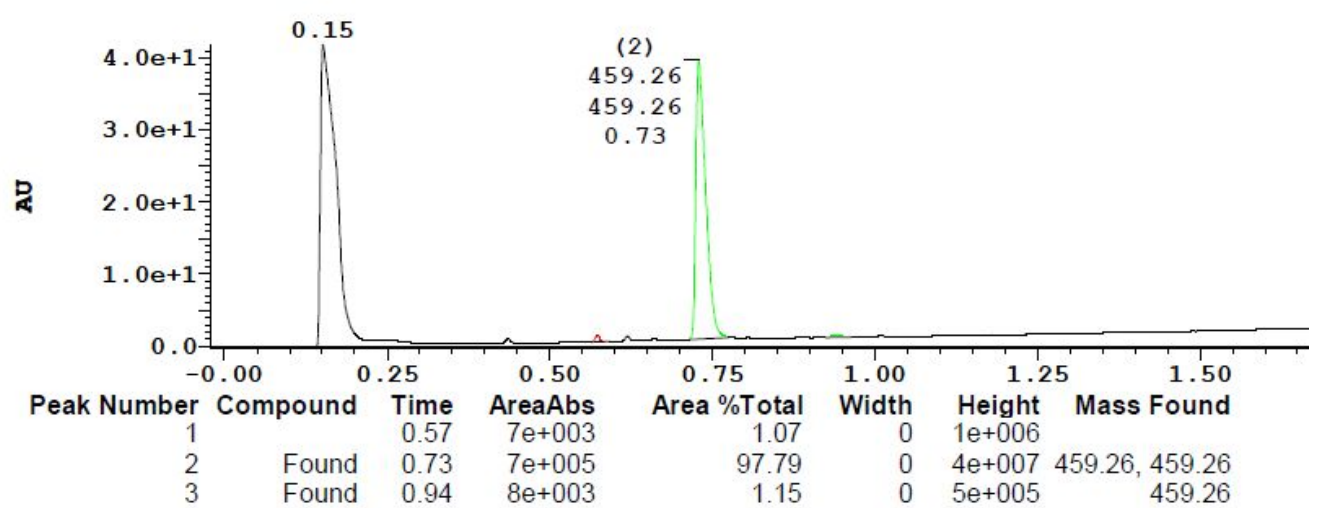


LCMS Cpd 28:

MS ES+ : $475.274 \quad 1.0000 \mathrm{Da}$

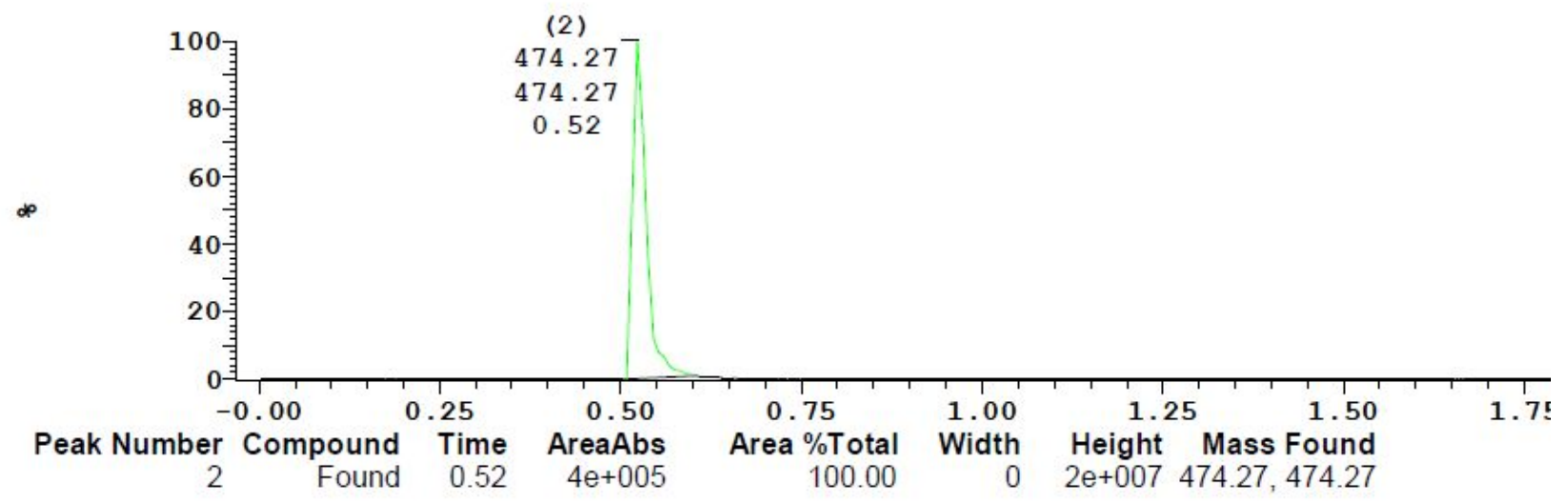

\section{LCMS Cpd 29:}

MS ES+ : 466.22 1.0000Da

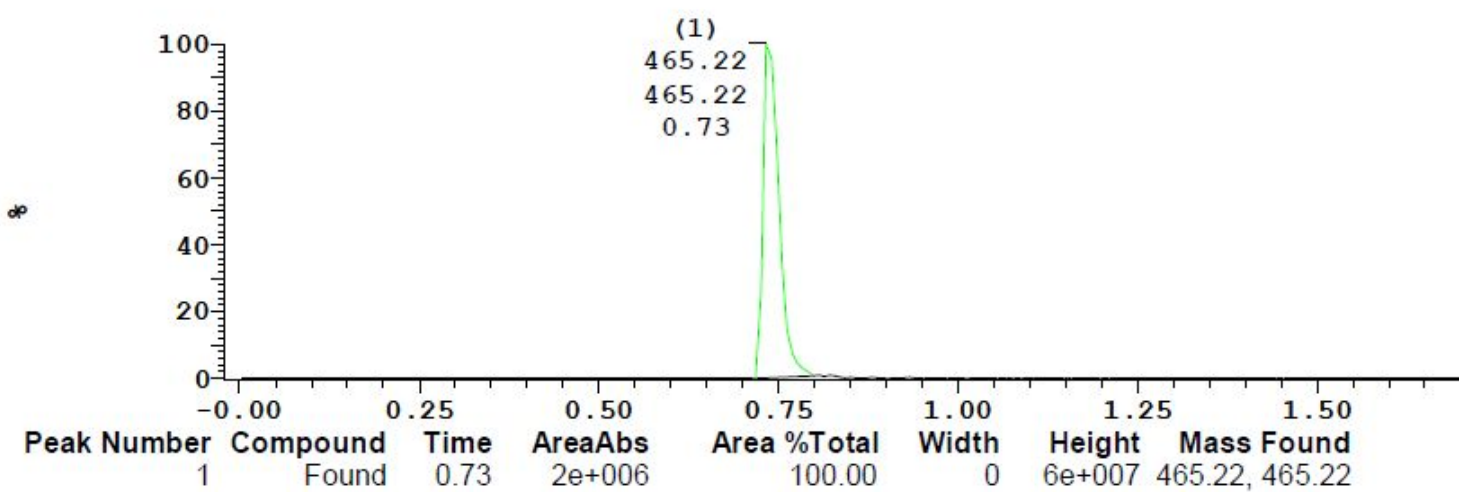




\section{LCMS Cpd 30:}

MS ES+ :466.22 $1.0000 \mathrm{Da}$

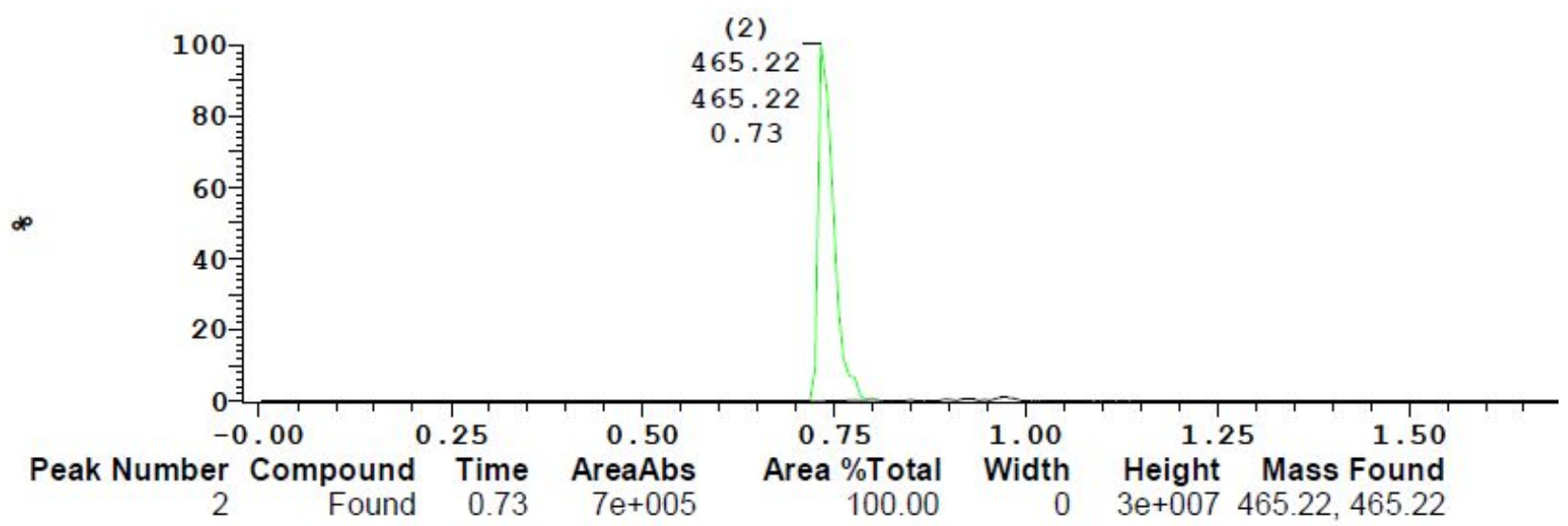

\section{LCMS Cpd 31:}

MS ES+ :450.243 $1.0000 \mathrm{Da}$

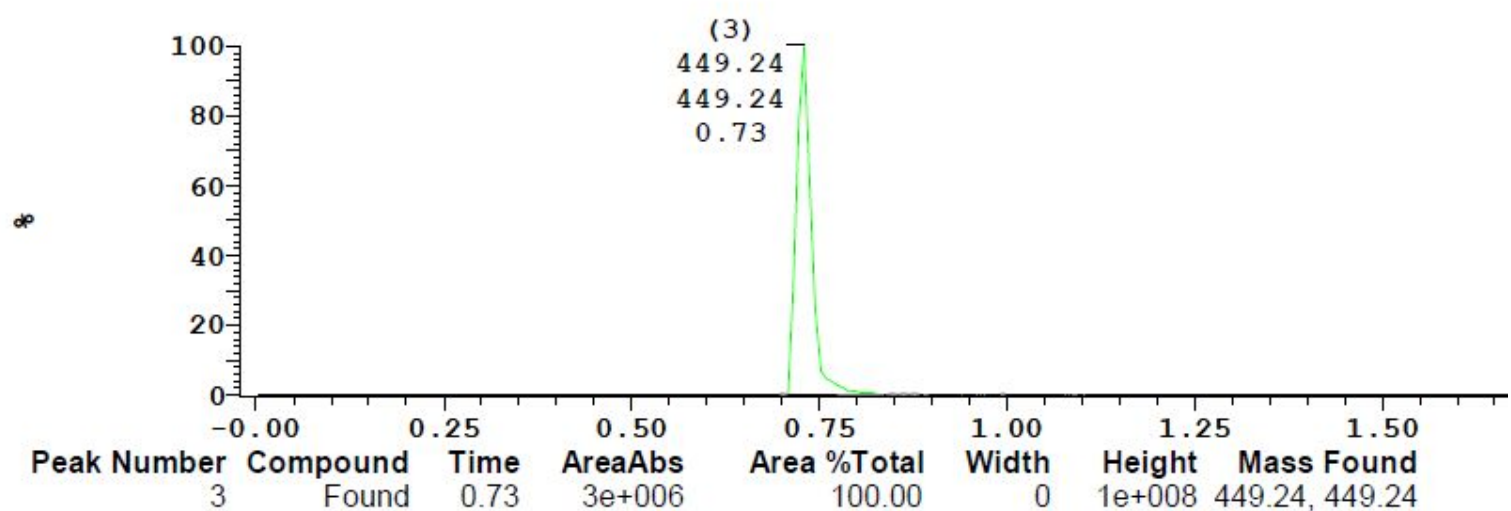




\section{LCMS Cpd 34:}

MS ES+ :450.243 1.0000Da

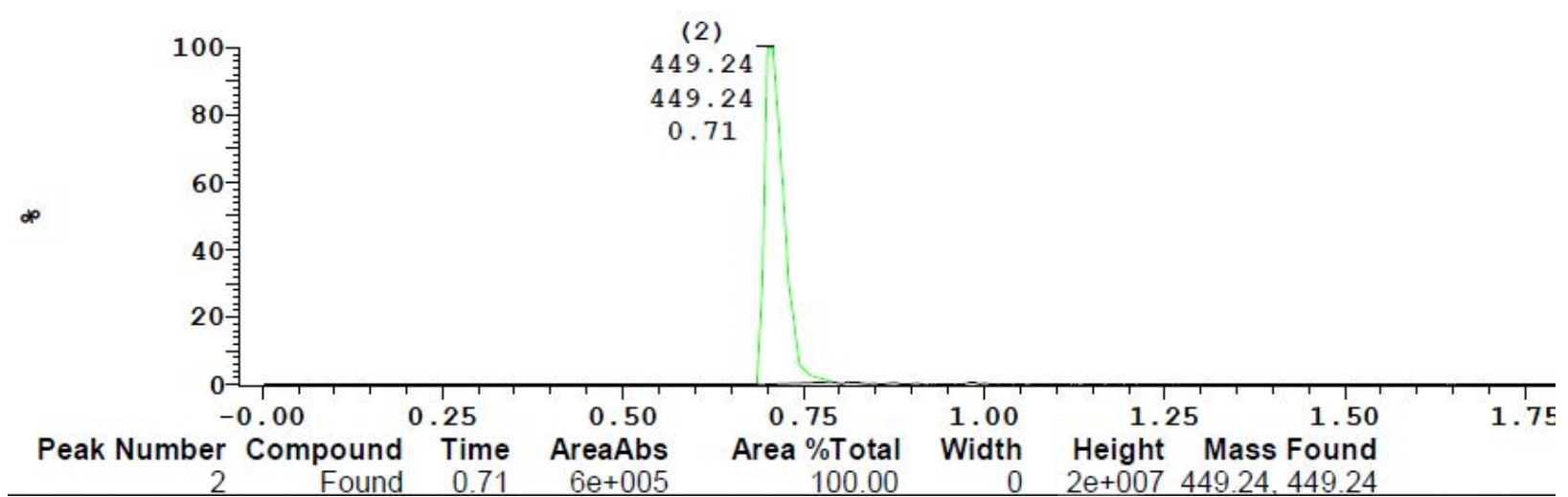

\section{LCMS Cpd 35:}

UV Detector: TIC

$$
\text { (1) }
$$

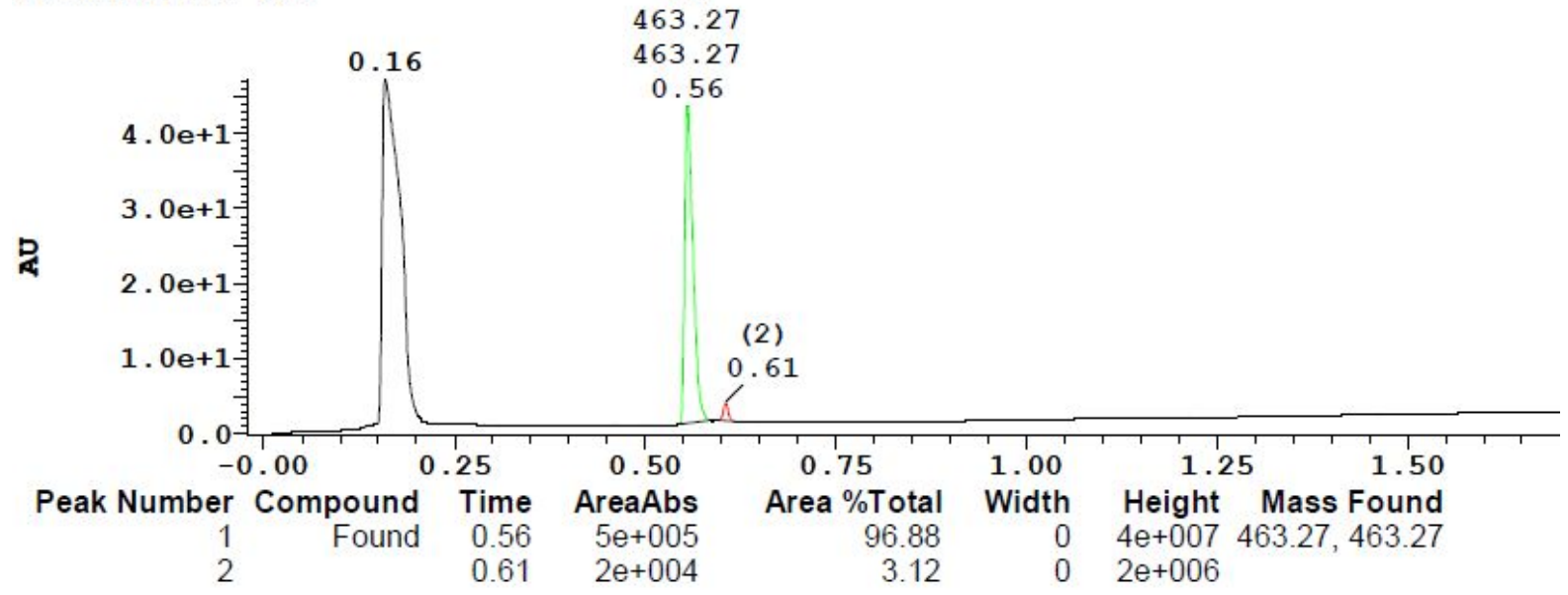




\section{LCMS Cpd 36:}

UV Detector: TIC

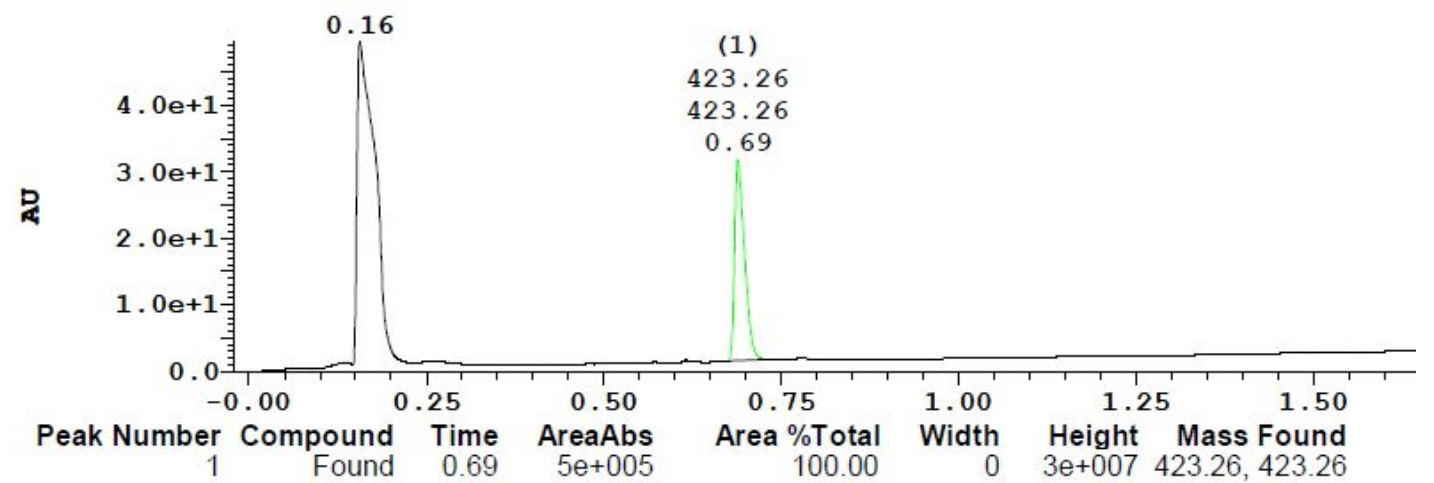

LCMS Cpd 37:

UV Detector: TIC

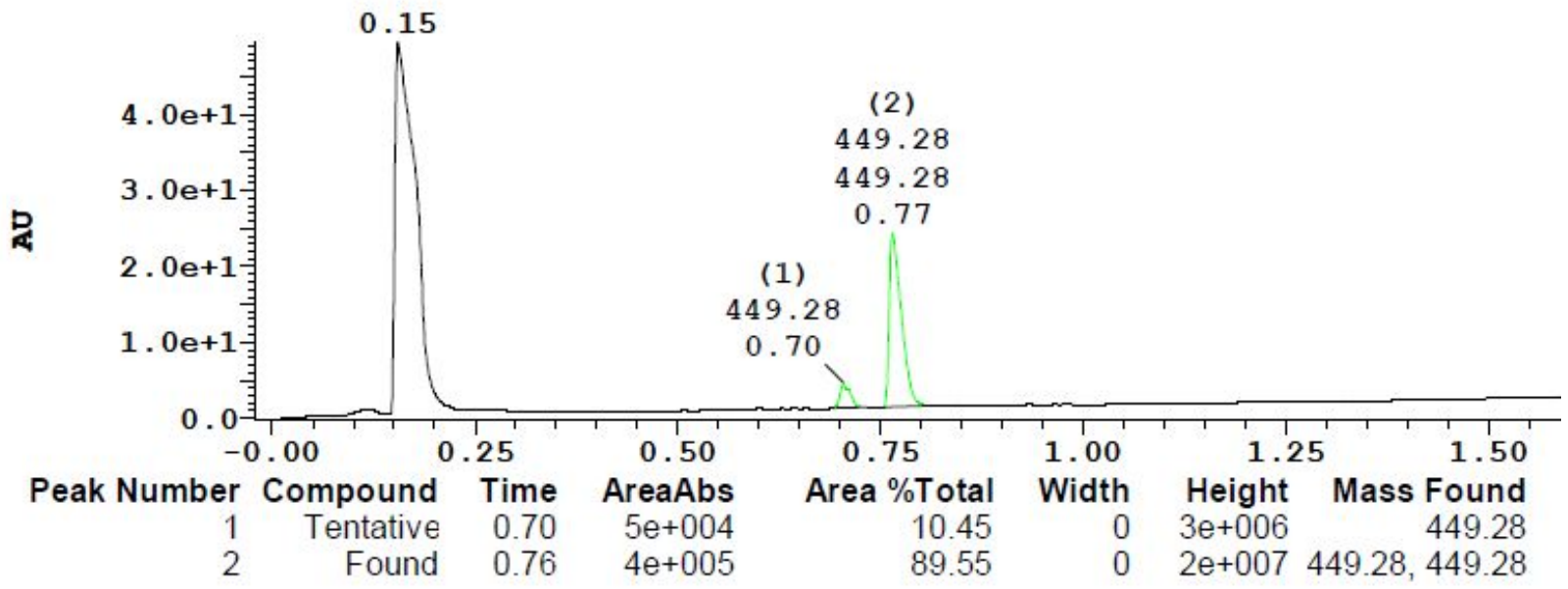


LCMS Cpd 38:

UV Detector: TIC

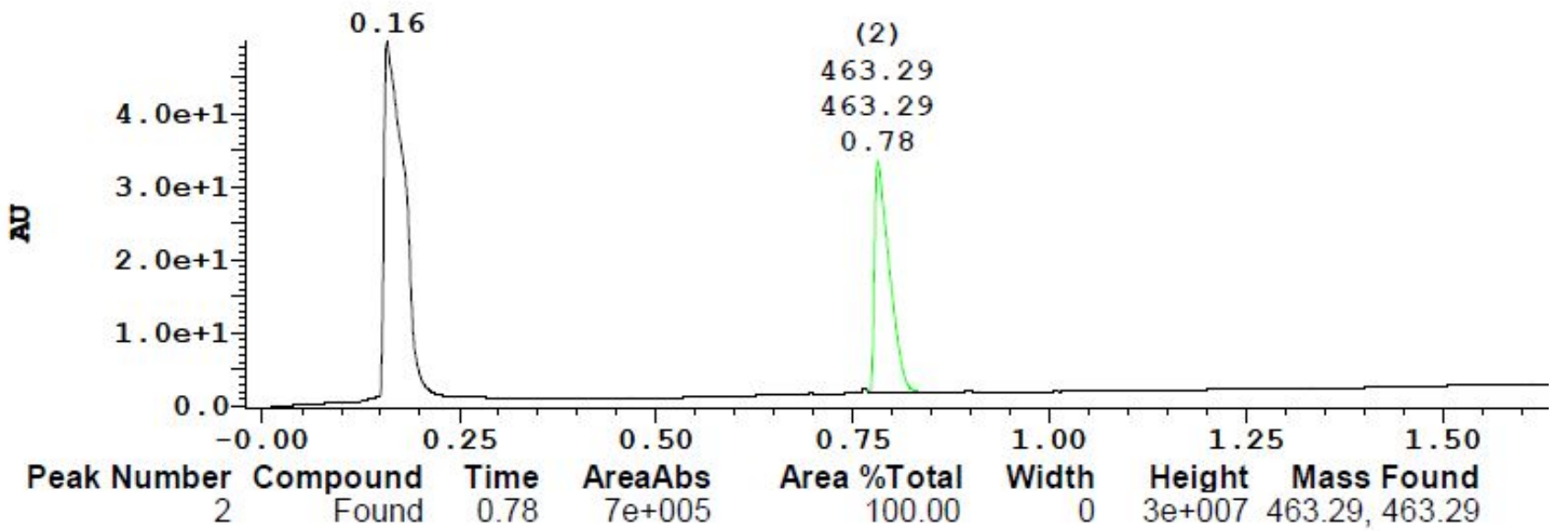

\section{LCMS Cpd 39:}

UV Detector: TIC

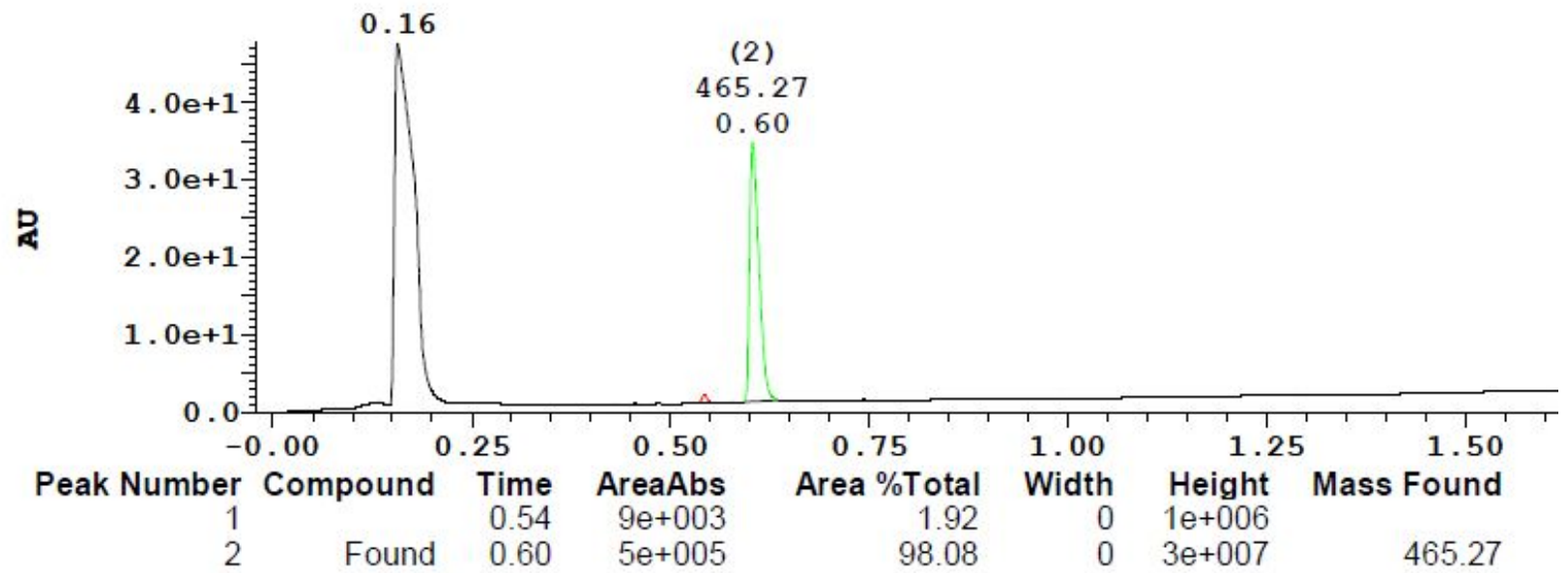




\section{LCMS Cpd 40:}

UV Detector: TIC

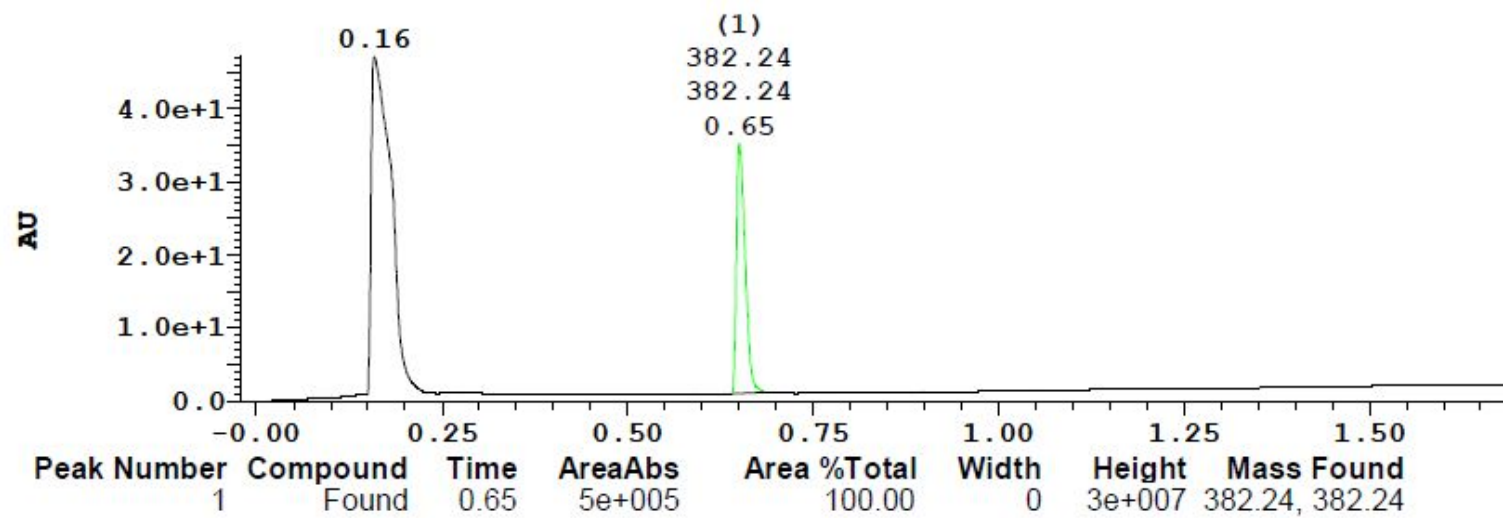

LCMS Cpd 41:

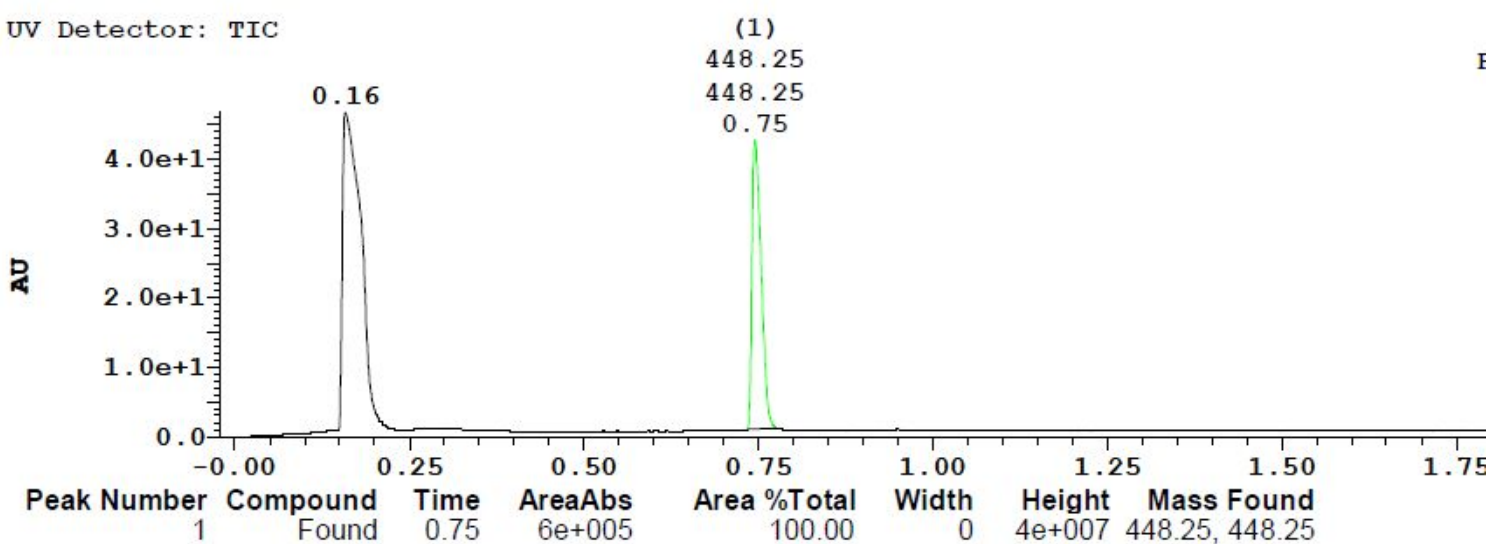




\section{LCMS Cpd 42:}

UV Detector: TIC

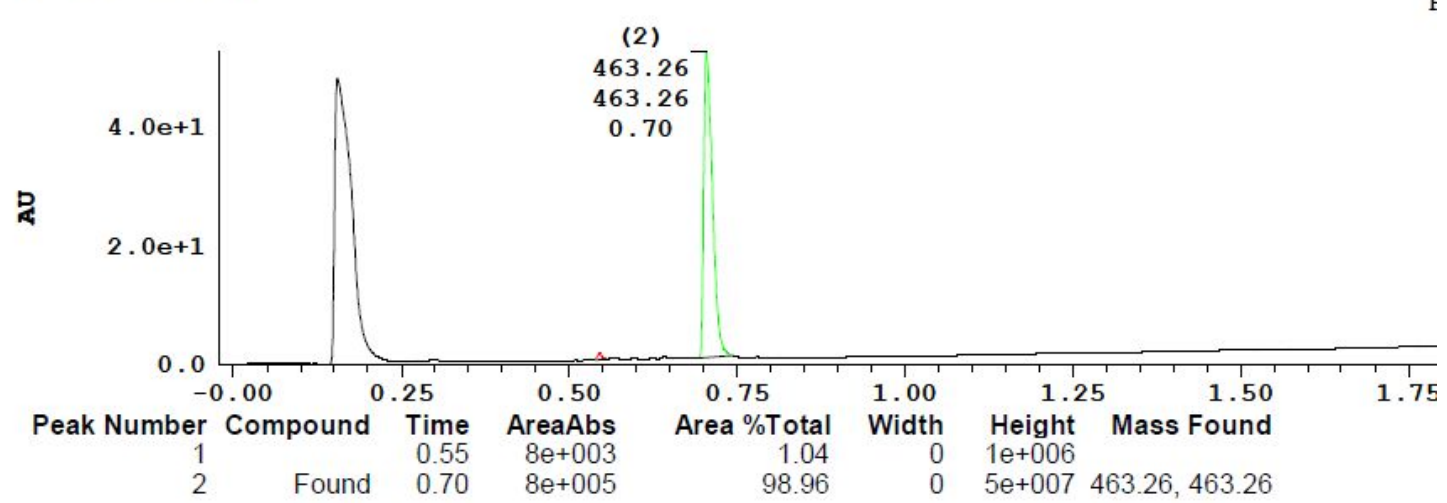

\section{LCMS Cpd 43:}

UV Detector: TIC

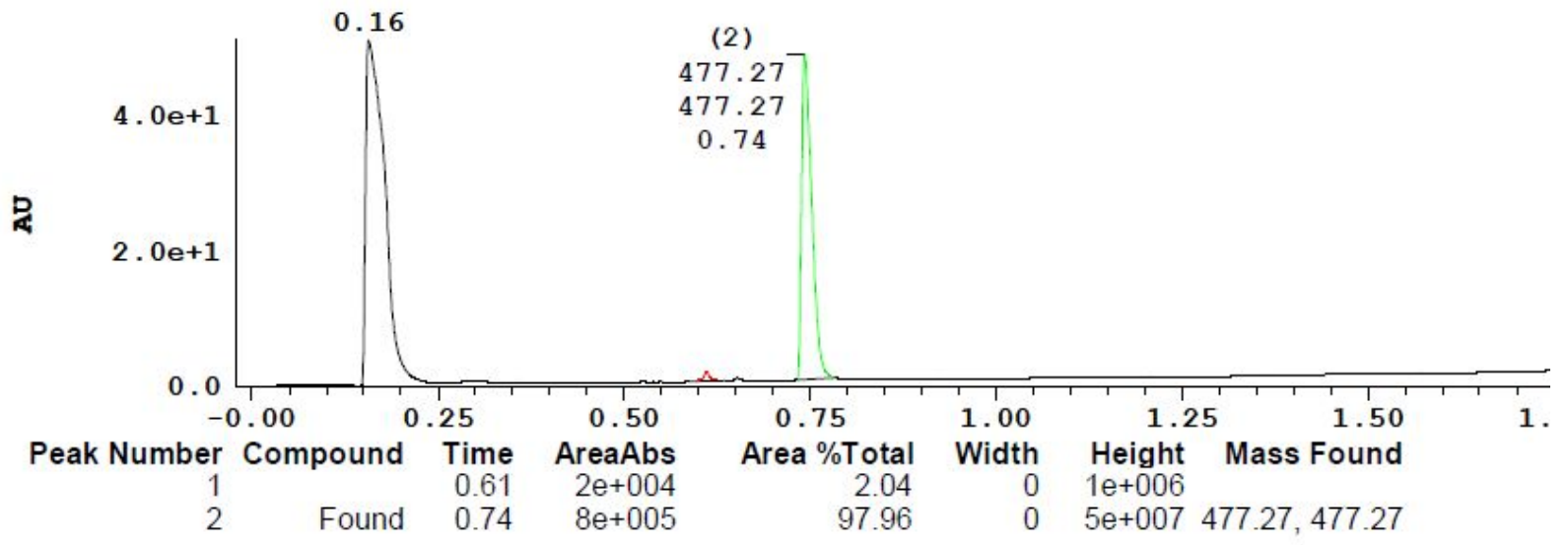




\section{LCMS Cpd 44:}

UV Detector: TIC

$$
\text { (2) }
$$

477.27

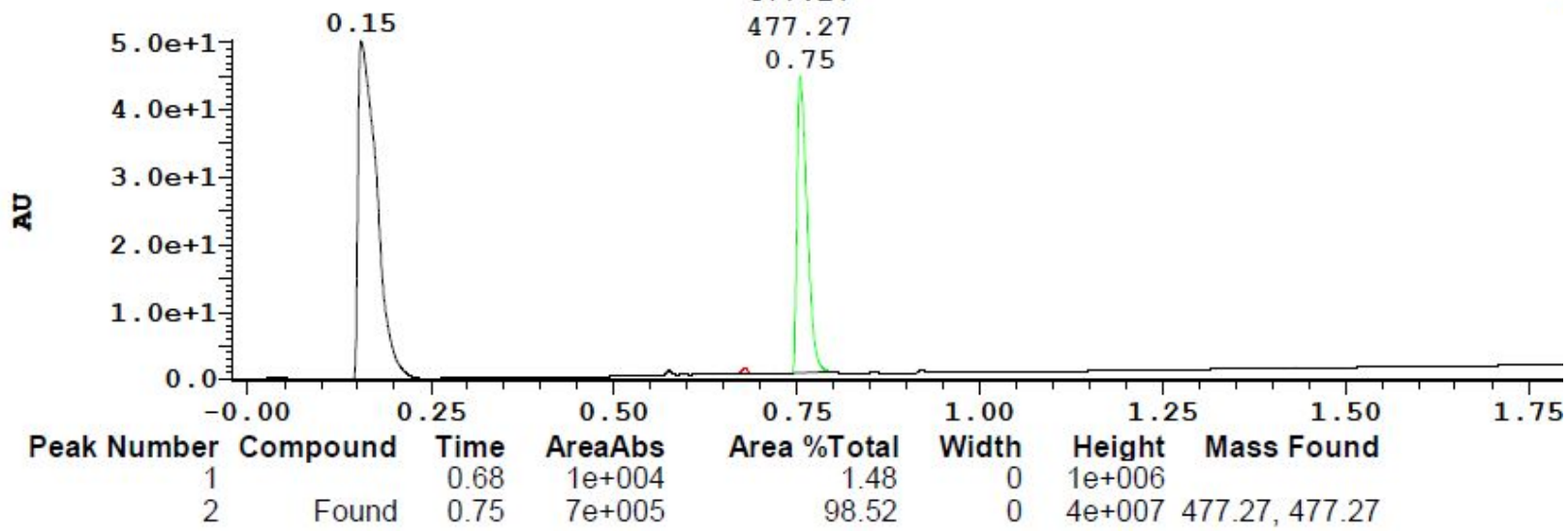

\section{LCMS Cpd 45:}

UV Detector: TIC

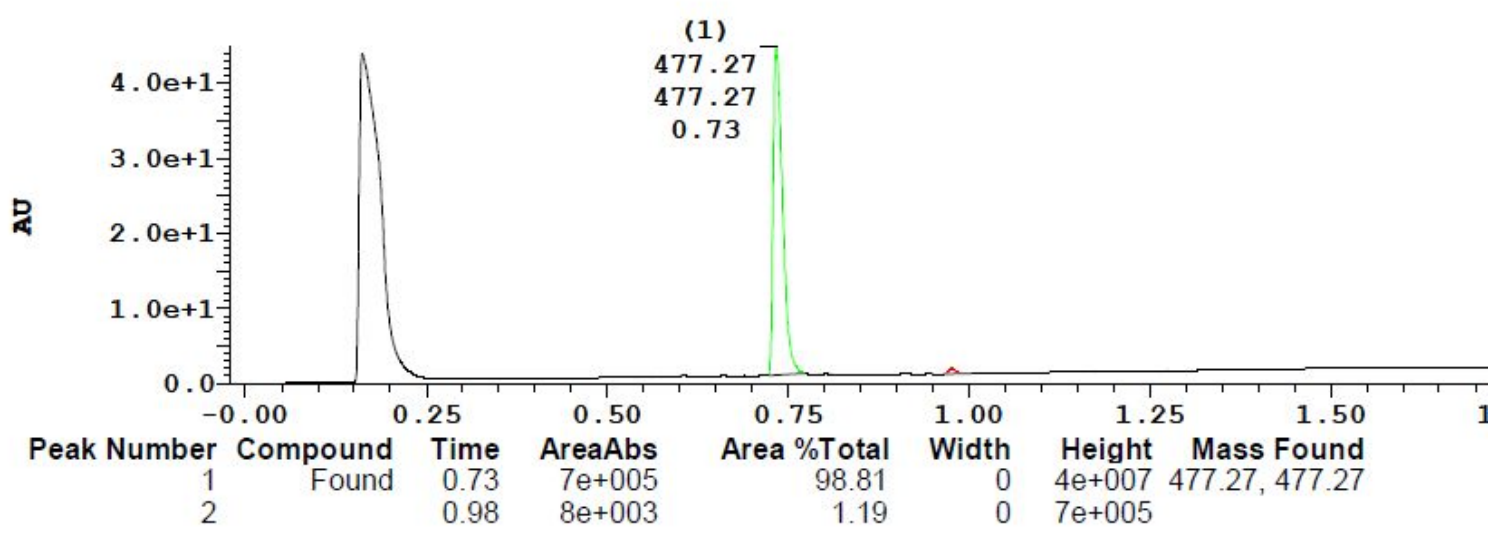




\section{LCMS Cpd 46:}

UV Detector: TIC (1)

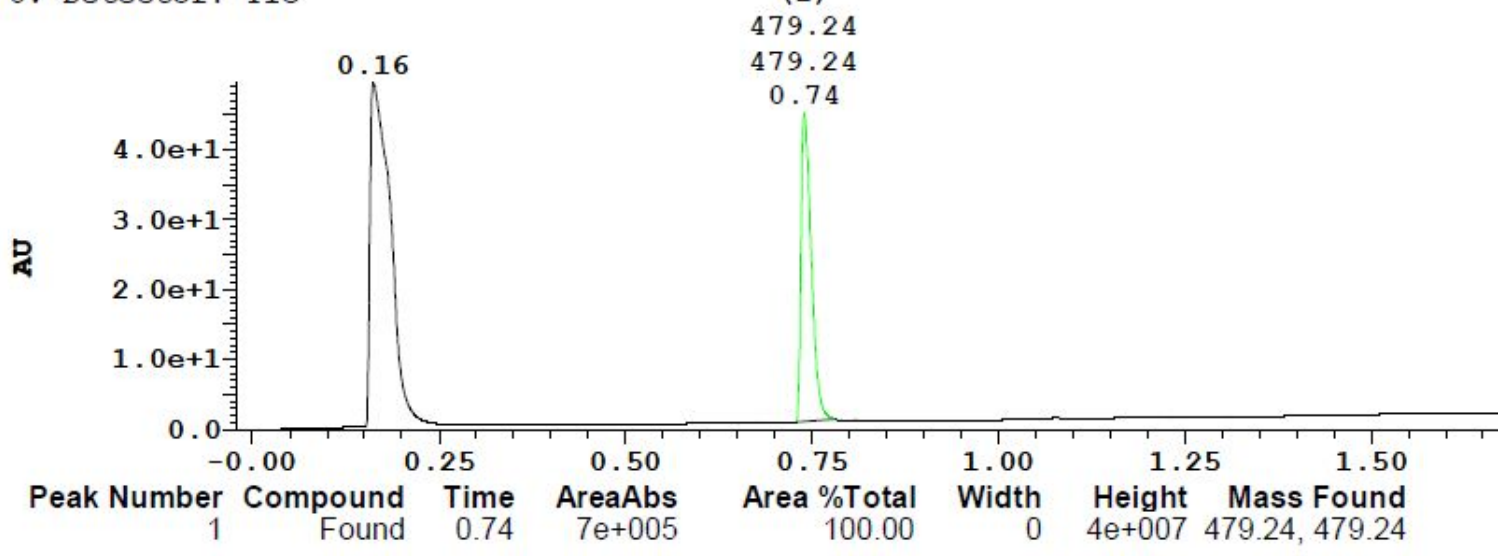

\section{LCMS Cpd 47:}

UV Detector: TIC

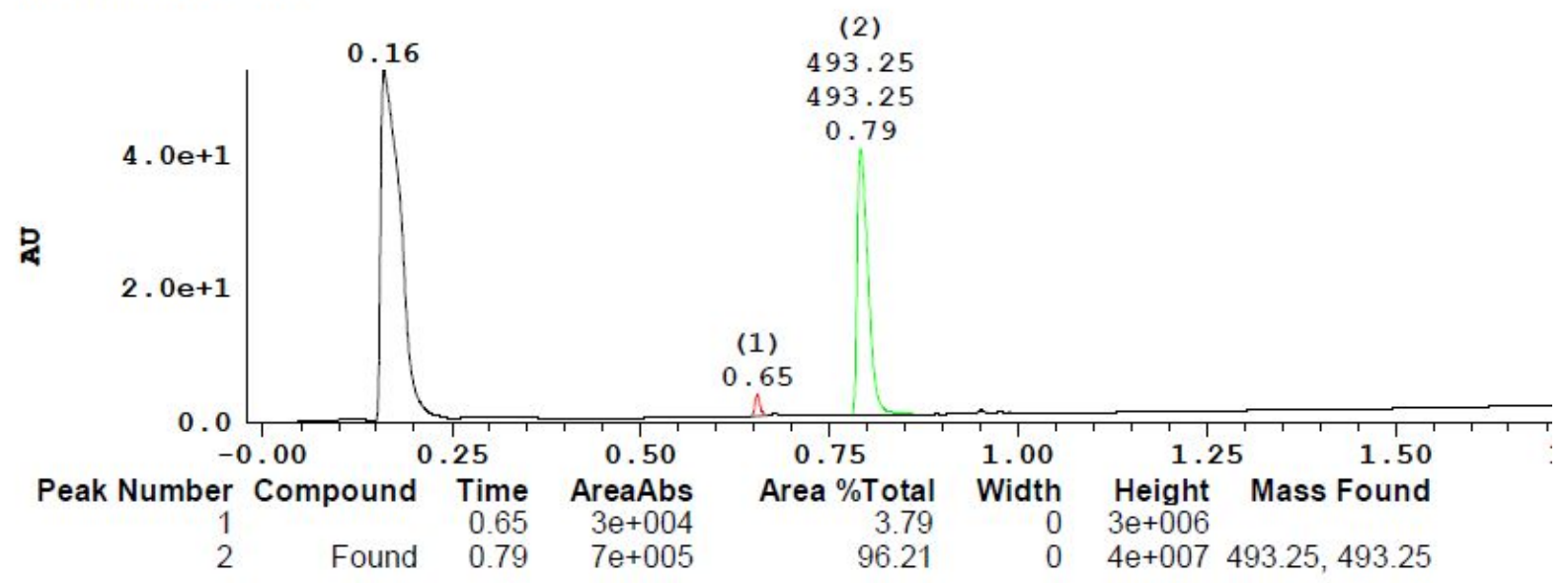




\section{LCMS Cpd 48:}

UV Detector: TIC

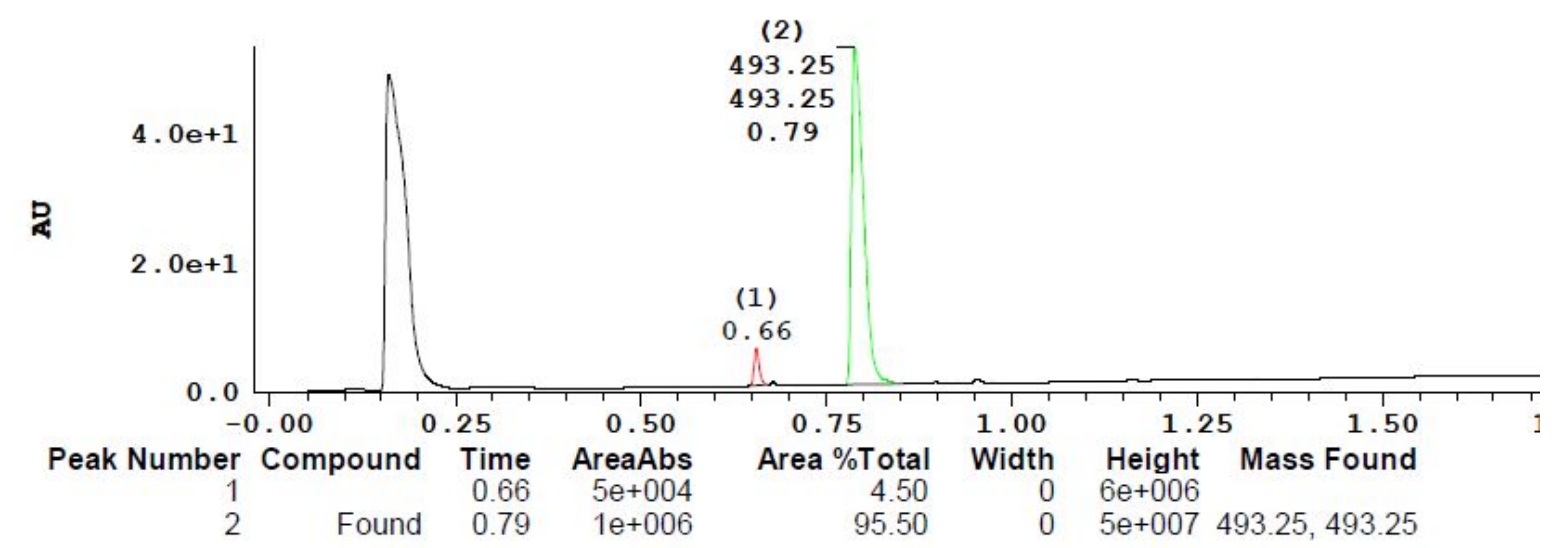

\section{LCMS Cpd 49:}

UV Detector: TIC

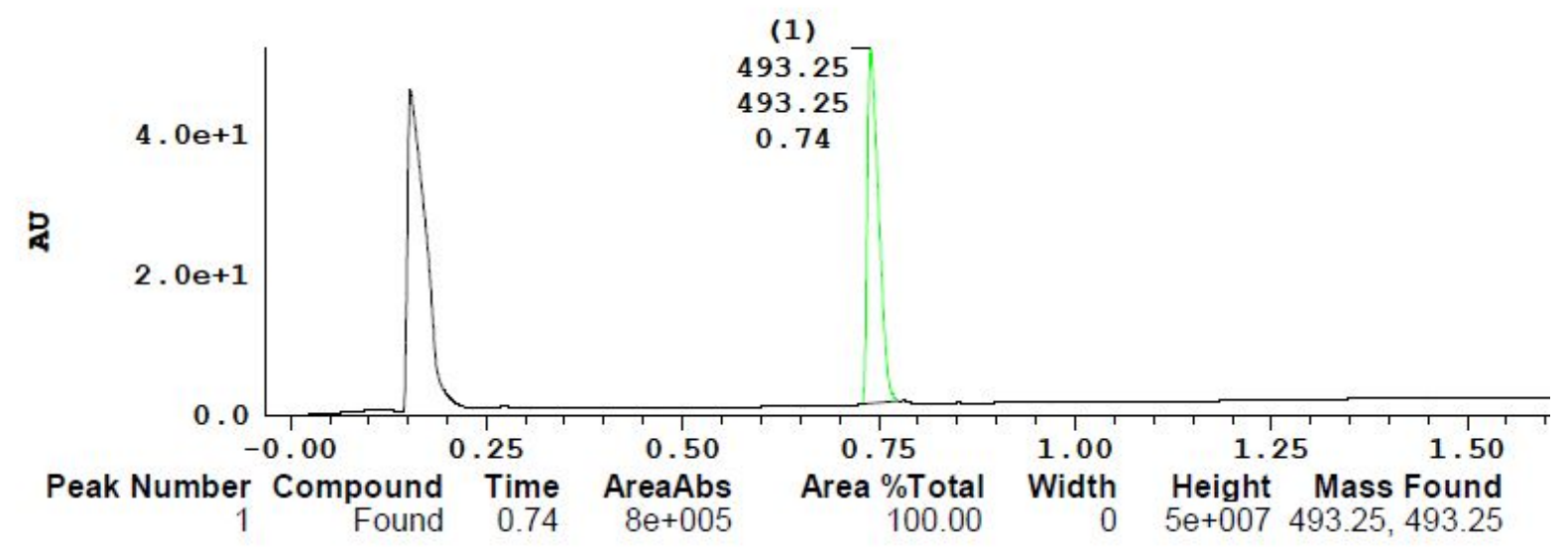


LCMS Cpd 50:

UV Detector: TIC

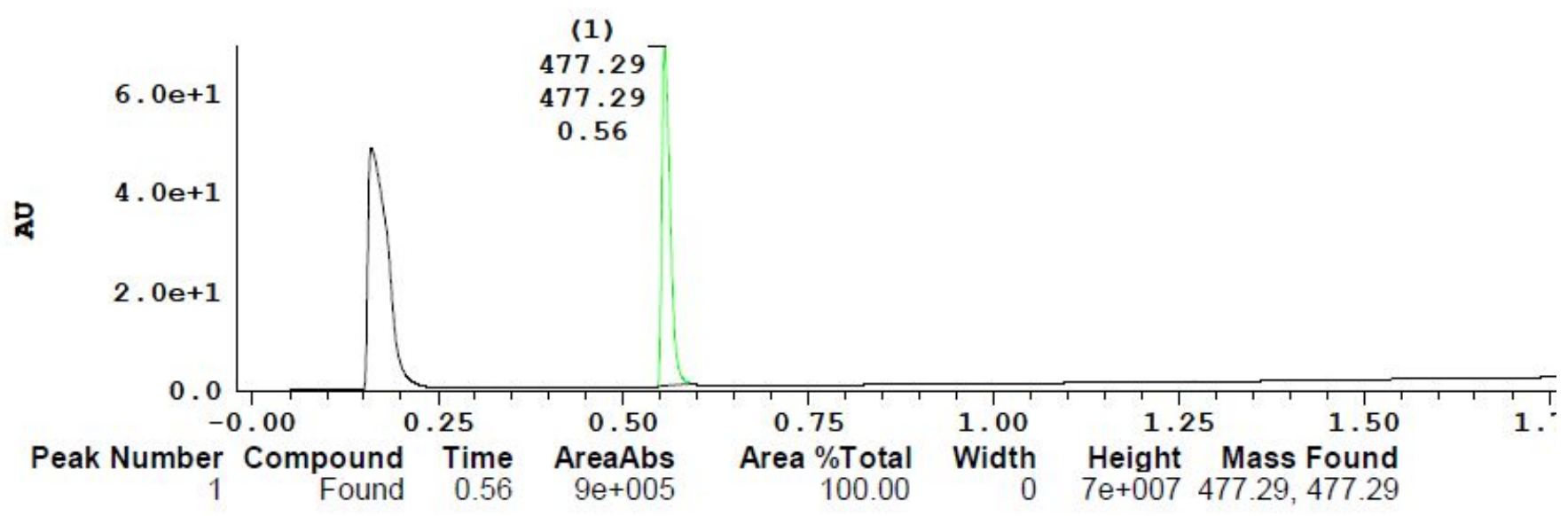

